\title{
Place et nécessité des travaux religieux, ethnohistoriques et linguistiques dans le paysage dictionnairique gabonais ${ }^{1}$
}

P.A. Mavoungou, Groupe de Recherches en Langues et Cultures Orales (GRELACO), Département des Sciences du Langage, Université Omar Bongo, Libreville, République Gabonaise (moudika2@yahoo.fr)

Résumé: Les dictionnaires devraient être le reflet d'une grande variété de découvertes enregistrées dans le domaine de la linguistique. Au Gabon, en ce qui concerne les rapports entre la lexicographie et la linguistique, des études doivent être menées. Cet article ne retrace pas seulement l'histoire de la discipline lexicographique au Gabon, mais également comment les travaux pionniers dans les domaines de la linguistique, de la religion et de l'ethnohistoire, ont servi (et devraient continuer de servir) de travaux de référence pour la compilation de nouveaux dictionnaires. Compte tenu du fait que la majorité des dictionnaires existant a été le résultat de la contribution de lexicographes isolés, on peut espérer que les futurs produits lexicographiques ne seront pas seulement des réservoirs de connaissances linguistiques et culturelles mais également le résultat d'un travail d'équipe.

Mots-clés: LEXICOGRAPHIE, UNITÉ(S)-LANGUE(S), LINGUISTIQUE, ÉTUDES RELIGIEUSES, ETHNOHISTOIRE, TRAVAIL D'ÉQUIPE, METALEXICOGRAPHIE

\begin{abstract}
The Place and Necessity of Religious, Ethnohistorical and Linguistic Works in the Gabonese Dictionary Landscape. Dictionaries should reflect a great diversity of results gained from research in the field of linguistics. In Gabon, as far as relationships between lexicography and linguistics are concerned, studies are still to be started. This article does not only trace the historical development of lexicography in Gabon, but also shows how earlier studies in the fields of linguistics, religion and ethnohistory have served (and should continue to serve) as reference works for the compilation of new dictionaries. Owing to the fact that most existing dictionaries have resulted from the enterprise of single lexicographers, it is hoped that future lexicographic products will not only be containers of linguistic and cultural knowledge but also the result of team efforts.
\end{abstract}

Keywords: LEXICOGRAPHY, LANGUAGE GROUP(S), LINGUISTICS, RELIGIOUS STUDIES, ETHNOHISTORY, TEAM WORK, METALEXICOGRAPHY

\section{Introduction et délimitation du sujet}

La liste plus ou moins complète des travaux sur les langues du Gabon a été établie (cf. Hombert et Mortier 1984, 1990). Il en est de même pour les travaux 
lexicographiques à proprement parler (cf. Nyangone Assam et Mavoungou 2000). Les rapports entre la lexicographie et la linguistique entre autres n'ont pas encore été abordés de façon systématique par la plupart des publications sur les langues du Gabon. La présente contribution a la prétention de combler un tant soit peu cette lacune.

Dans cet aperçu des travaux sur les langues gabonaises, nous nous sommes imposés les restrictions suivantes:

(a) Le foisonnement relatif des travaux ethnohistoriques, religieux et linguistiques sur les langues gabonaises, nous contraint dans la plupart des cas à une simple énumération.

(b) Nous excluons de cet aperçu tous les dictionnaires plurilingues ou multilingues (c'est-à-dire trois langues et plus).

(c) Parmi les dictionnaires bilingues (puisque c'est d'eux qu'il s'agit dans cet aperçu), nous ne parlerons pas des dictionnaires des langues de spécialités.

L'état des lieux sur les travaux sur les langues du Gabon se fera selon les dix unités-langues de Kwenzi-Mikala $(1988,1998)$. Le traitement de chaque unitélangue fera apparaître çà et là des disproportions que le lecteur avisé ne manquera pas de noter. En effet, le développement est fonction de la littérature disponible sur chaque groupe.

\section{Le Groupe Mazuna}

Les parlers de ce groupe se localisent principalement dans trois des neuf provinces du Gabon, à savoir: l'Estuaire, l'Ogooué-Ivindo et le Woleu-Ntem. Le fan-ntumu est parlé à Oyem et Bitam, et le fan-okak à Médouneu, Cocobeach et Mitzic. Le fan-atsi est parlé à Ndjolé et à Lambaréné, le fan-make à Libreville et Kango, le fan-mvaï se retrouve à Minvoul, et le fan-nzaman est parlé dans la région de Makokou-Ovan-Booué. Le fan est une langue transfrontalière parce qu'il est également parlé dans les pays voisins, à savoir en Guinée-Équatoriale, au Cameroun, à São Tomé et Principé (Grimes 1996) et en République du Congo.

Tous les travaux disponibles en littérature mazuna touchent les domaines de la religion, l'ethnohistoire, la linguistique et la lexicographie. Dans cette section, nous mettrons beaucoup plus l'accent sur les travaux ethnohistoriques et linguistiques avant de présenter quelques études lexicographiques.

Comme cela apparaît dans la localisation ci-dessus, le fan est divisé en plusieurs dialectes. Le make est unanimement reconnu comme le dialecte du fan le plus abondamment documenté en matière religieuse (Afane Otsaga 2004). Ceci tient notamment à une situation ou position géographique stratégique. En effet, disséminées dans la province de l'Estuaire (Libreville, Kango, 
Ntoum et Foulenzem), les populations Fan-Make sont très tôt rentrées en contact avec les explorateurs européens et les colons français. Ces derniers seront très tôt confrontés au problème de la communication avec les populations autochtones. Afin de surmonter les barrières linguistiques, les missionnaires, notamment, vont se lancer dans la production de catéchismes, de recueils de cantiques, des traductions d'évangiles et des manuels scolaires pour établir leur oeuvre d'évangélisation et de scolarisation. Cette production religieuse et scolaire intervient sous les auspices de la Mission Catholique de Libreville (Vicariat Apostolique de Libreville). Déjà depuis le Concile de Trente (1545-1563), les prêtres devaient apprendre les langues de leurs futurs convertis, non seulement pour propager le christianisme, mais aussi parce que cela favorisait l'imposition. Pour la langue fan, les missionnaires impliqués dans la traduction de la Bible et des Évangiles la faisaient en fan-make, le dialecte fan le plus usité au siège du clergé catholique du Gabon (la Mission Catholique de Libreville).

L'ouvrage qui fait autorité depuis sa parution en 1962 est la traduction de la Bible en fan par François Ndong. Cette traduction intitulé Nten Wam est évidemment rédigée en fan-make , langue maternelle de l'archevêque Ndong. Dans le contexte des dispositions du Concile de Trente qui avait encouragé l'évangélisation dans les langues vernaculaires, monseigneur Ndong va également jouer un rôle instrumental dans la production de nombre de travaux dans le dialecte make (Afane Otsaga 2004).

Pour ce qui est des traductions des Évangiles, citons: Évangile de Jean: traduction dans la langue des Fan (1910), Évangile de Luc: traduction dans la langue des Fan (1910), et Évangile de Matthieu: traduction dans la langue des Fan (1902). À ces Évangiles selon Jean, Luc et Matthieu, il faut ajouter non seulement des livres de prières (Livre de prières = NTEN WAM minkobe ma nè ge kobe né nzame 1898), des livres de questions ou catéchismes (Catéchisme Fan 1932, Katesism Nten wa vegele Fang Nsong Katolik = Catéchisme de la Doctrine Catholique n.d., et Nteni osi nteni Fañwe 1889) mais également des ouvrages qui sont beaucoup plus des objets scolaires que des travaux religieux: Syllabaire Fan (1925), Ntèn wa tar'éyé gele fang'ne fala étén nélang = Exercices de lecture et d'écriture en pahouin et en français (1898).

Tous ces travaux aussi bien liturgiques que bibliques en fan-make constituent une matière lexicographique non négligeable. Une fois intégrés, par exemple au moyen de la méthode de l'OCR (Optical Character Recognition) ou de la saisie, ces textes monolingues pourraient être renforcés par des données orales collectées sur le terrain. On pourrait ainsi obtenir à terme de larges bases de données textuelles électroniques ou corpora en fan-make notamment. Plusieurs types de dictionnaires (monolingues, bilingues ou multilingues) pourraient être dérivés à partir desdits corpora électroniques.

Par ailleurs, les syllabaires et abécédaires disponibles en fan sont, en quelque sorte, des dictionnaires de prononciation associant objets et lettres (voire sons). On dispose ainsi d'extraits textuels à trois colonnes disposées par séries de consonnes en variations sur une voyelle. Le lecteur aura une idée du type de 
répertoire dont il s'agit d'après le bref fragment suivant, qui présente une série de consonnes en variations sur la voyelle /a/; dans la première colonne apparaissent une série d'illustrations éminemment didactiques. La deuxième colonne rend compte de l'association des objets et des lettres (et même des sons). En effet, les graphèmes (voire syllabogrammes: signes à valeur "phonétique") apparaissent avec à l'arrière plan les dessins les illustrant. Enfin la troisième colonne correspond aux traductions fan en graphèmes voire syllabogrammes des deux premières colonnes.

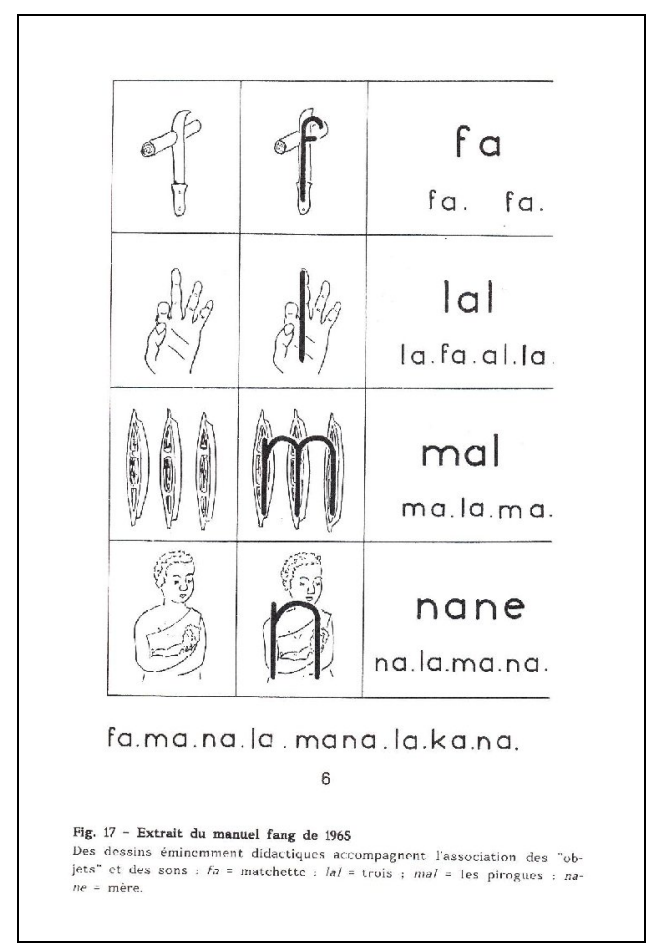

Figure 1: Quelques syllabogrammes du fan sur fond d'illustrations (extrait de l'ouvrage Ma yi lañ nten fañ osua (1965) publié sous les auspices de l'Église Évangélique du Gabon, tel que cité dans Mayer (1990: 86)

Ces productions religieuses et scolaires ont également le mérite de fixer un premier état de la langue, en particulier une orthographe jusque là très fluctuante. En outre, la plupart de ces travaux comportent un certain nombre de lacunes qui doivent être comblées. Par exemple, ils ne marquent pas les tons ainsi que la prononciation de la langue. La lacune la plus importante et la plus souvent citée est que ces travaux contiennent beaucoup d'inconsistances orthographiques. Par exemple, Alexandre (1961) mentionne que la langue fan a acquis "trois transcriptions [catholiques] et deux [protestantes] pour environ un million de locuteurs, sans parler des différences morphologiques dues à des 
calques catholiques sur l'allemand, le français et l'espagnol, et protestants, sur l'américain".

Les premiers travaux à caractère ethnohistorique sur le Fan abordent essentiellement la question des migrations. Les récits migratoires sont ponctués d'épisodes guerriers où se côtoient l'imaginaire, l'extraordinaire et le mystique. Par conséquent et d'une manière générale, le récit des migrations des Fan contient aussi bien des éléments ou données mythiques qu'historiques. Le récit mythique fait partir le peuple Fan d'un lieu difficile à identifier de nos jours appelé "Odzamboga". C'est au cours de leur déplacement que les populations Fan seront confrontées à une épreuve physique de taille. En effet, elles vont tomber nez à nez avec un arbre gigantesque appelé "Adzap". Ne pouvant ni contourner ni passer au-dessus de l'"Adzap", les populations n'avaient pas d'autre choix que de pratiquer une ouverture dans l'arbre. Ainsi va naître et se cristalliser dans la mémoire collective la légende du "trou de l'Adzap". Traverser l'Adzap" de part en part n'était pas chose facile, les Fan y arrivèrent au prix de dures et tenaces efforts. C'est seulement après la rencontre et le franchissement de cet obstacle naturel que ces populations vont se retrouver disséminées à des proportions diverses dans les provinces du Gabon: Estuaire, MoyenOgooué, Ogooué-Ivindo et Woleu-Ntem.

D'un point de vue historique, les migrations du peuple Fan remontent approximativement aux 18ème et 19ème siècles. Les régions du Dja, l'Ivindo et le Ntem sont considérées comme les dernières étapes avant la dispersion des populations Fan (Mouguiama-Daouda 1995). Selon un certain nombre d'auteurs (Alexandre 1967 et Balandier 1957, 1963 entre autres), c'est notamment sous la pression des Foulbé que les Fan à partir du Cameroun vont atteindre le Moyen-Ogooué au début du 19ième siècle. Leur arrivée dans la province de l'Estuaire pourrait se situer autour de 1860. Les superlatifs ne manquent pas pour qualifier ces nouveaux venus, "une belle race [...] des hommes grands, bien faits" (le Marquis de Compiègne, cité par Pierre Alexandre 1967). Tous ces récits sur les migrations des Fan constituent de facto le matériel de base pour la rédaction des notes introductives traitant des questions ethnohistoriques dans les dictionnaires en fan.

D'un point de vue purement linguistique, le terme fan désigne aussi bien la langue que ses locuteurs. Il s'agit d'une langue bantou de la zone A70, avec plus précisément le sigle A75 selon la classification de Guthrie (1948, 1953 et 1969-1971). En outre, le glossonyme et ethnonyme Pahouin, écrit selon une orthographe administrative française tronquée, est souvent utilisé pour désigner les Fan. Les travaux linguistiques en fan sont exogènes. Les travaux de Marling (1872), Martrou (1924), etc. sont certes des travaux lexicographiques mais ils sont précédés d'une description grammaticale du fan. Mis à part les travaux de Raponda-Walker notamment, c'est avec l'ouverture à l'Université Omar Bongo d'une option linguistique au sein du Département des Lettres Modernes que l'on va constater que de plus en plus de travaux linguistiques sur les langues locales seront menés par les Gabonais eux-mêmes. Nombre de travaux linguistiques portant sur le fan ont été présentés et soutenus à l'Uni- 
versité Omar Bongo ou faits par des étudiants gabonais dans les universités européennes. Il s'agit pour la plupart des Rapports de Licence, Mémoires de Maîtrise et Thèses de Doctorat. Citons: Mbougou Asseko (1979), Ndong Menini (1980), Mba-Nzué (1981), Ondo Essono Aba'a (1981), Mba-Nkoghe (1981), Andeme Allogho (1983), Nzang-Bié (1986), Rekanga (1986), Nguema Nzue (1988), Cinnamon (1990) et Ondo-Mebiame (1992). Les travaux de Ndong Menini, Ondo Essono Aba'a, Nzang-Bié, Rekanga, Nguema Nzue et Ondo-Mebiame décrivent le fan-ntumu. Mba-Nzué propose une description du fanmvaï, tandis qu'Andeme Allogho et Cinnamon décrivent le fan-nzaman. Les données utilisées dans l'étude menée par Mba-Nkoghe sur la phonologie et la morphologie proviennent du fan-atsi parlé dans les zones de Ndjolé et Lambaréné. Le travail de Mbougou Asseko qui est une étude contrastive entre le fan d'Oyem et celui de Libreville est un peu à l'image de ce qui se fait actuellement et cela depuis 1994 au Département des Sciences du Langage de l'Université Omar Bongo. Nombre de travaux linguistiques en fan soutenus dans ledit Département abordent la langue fan sous l'angle de la comparaison avec notamment le français, la langue officielle au Gabon (sur le statut du français au Gabon ainsi que la place et l'utilité des langues gabonaises dans l'activité nationale, lire Mba-Nkoghe 1991). Citons par exemple: Mekui Missang (1998), Ella Ella (2000), Ella (2000) et Bibang Meye (2001). Enfin, il convient de signaler que les travaux susmentionnés sur le fan concernent la perspective synchronique. Par contre, la contribution de Medjo-Mvé (1997) envisage la perspective historique du fan.

Tous ces travaux linguistiques sur le fan constituent une matière lexicographique non négligeable. En effet, ils présentent en majorité des corpora plus ou moins étendus qui pourraient être énormément enrichis afin de répondre aux normes dictionnairiques.

Sur le plan lexicographique stricto sensu, le premier dictionnaire décrivant le fan est celui de Marling, publié en 1872 à New York. La contribution de Marling intitulée Dictionnaire fang-français est suivie en 1881 d'un lexique Panze Primer and Vocabulary compilé par Nassau à partir du matériau collecté par H.M. Adam. Ce travail lexicographique est précédé d'une description grammaticale de la langue décrite. La comparaison entre ces deux travaux lexicographiques révèle que dans le travail de Marling, le fan est la langue source tandis que le français est la langue cible. Ces langues paires sont inversées dans le travail de Lejeune (1892). Du point de vue de la portée, ces deux dictionnaires ont un dénominateur commun: ce sont des ouvrages monocopals et tous d'eux décrivent le dialecte fan-atsi. Un dictionnaire monoscopal est une publication incluant seulement une section traitant la langue source et la langue cible $(\mathrm{A}>\mathrm{B})$. En d'autres termes, c'est une publication dans laquelle le lexicographe traite uniquement une langue source et une langue cible. Par exemple: le dictionnaire monoscopal de Marling a le fan comme langue source et le français comme langue cible. Ceci implique que seuls les items lexicaux d'un membre du couple de langues (le fan) seront lemmatisés. Par contre, dans un ouvrage biscopal, le lexicographe travaillera avec deux langues sources et deux langues 
cibles, c'est-à-dire qu'il inclura à la fois des sections $\mathrm{A}>\mathrm{B}$ et $\mathrm{B}>\mathrm{A}$ dans le même dictionnaire. À la suite des travaux de Marling et Lejeune, l'autre ouvrage lexicographique d'importance est celui de Largeau (1901). Il s'agit de l'Encyclopédie pahouine. D'un point de vue typologique, les ouvrages de référence lexicographique connaissent généralement une présentation tripartite ou trigonométrique: dictionnaire, encyclopédie et dictionnaire encyclopédique. L'Encyclopédie pahouine de Largeau est en réalité un dictionnaire encyclopédique. Il contient notamment des principes grammaticaux et une nomenclature français-pahouin. Les lemmes dans une encyclopédie sont classés par ordre thématique. Or, dans la nomenclature français-pahouin de Largeau, les unités de traitement apparaissent dans un ordre strictement alphabétique.

À la suite du travail de Largeau, l'autre ouvrage lexicographique qui mérite d'être mentionné est le bilingue Lexique fãn-français produit par monseigneur Martrou (1924), un missionnaire de la Congrégation des Pères du Saint-Esprit. Bien que le titre de cet ouvrage soit modestement décrit comme un "lexique", il s'agit en réalité d'un dictionnaire de volume réduit comprenant 3431 articles et couvrant 137 pages. Sur le plan microstructurel, ce travail lexicographique fournit aux usagers une variété de catégories de données, à savoir: la paraphrase du sens, les traductions, les exemples d'emploi, etc. (Mavoungou 2001: 127-130 et Mavoungou, Afane Otsaga et Mihindou 2003: 136).

Après la publication du dictionnaire de Largeau, l'activité de la lexicographie du fan-atsi va connaître un ralentissement. Cette activité va reprendre à la faveur de l'accession du Gabon à la souveraineté internationale. C'est précisément quatre ans (en 1964) après les Indépendances que le Dictionnaire fangfrançais et français-fang est publié sous les auspices de l'Association Française des Amis d'Albert Schweitzer. Cette publication posthume du pasteur genevois Samuel Galley contient deux parties: le dictionnaire à proprement parler suivi d'une grammaire fan.

Le dernier travail en lexicographie fan qui mérite d'être cité concerne un travail de métalexicographie (recherche sur les dictionnaires). Il s'agit de l'Esquisse d'un dictionnaire fang-mekè. Ce travail mené par Nzang-Obame (2004) constitue une espèce de rupture épistémologique parce qu'il présente un modèle théorique d'un dictionnaire en fan-make. L'étude se donne notamment pour objectif de concevoir l'ensemble des structures dudit dictionnaire complété par quelques échantillons d'articles traités. Pour les ouvrages lexicographiques qui l'ont précédé, on peut arguer qu'à un degré plus ou moindre, ils manquent d'une base métalexicographique. Toutefois, ces manquements sont excusables si l'on considère que les lexicographes de ces travaux précurseurs n'étaient ni linguistes, ni lexicographes qualifiés. Dans le même esprit que le travail de Nzang-Obame (2001), Afane Otsaga (2004) propose de A à Z la démarche pour la compilation et la publication d'un dictionnaire standard du fan. Concrètement, l'auteur analyse un certain nombre de critères scientifiques susceptibles de permettre qu'un des six dialectes du fan soit érigé ou élevé au rang de variante standard. C'est ce dialecte du fan susceptible de jouer le rôle de langue standard qui bénéficiera de l'ensemble des avantages liés à l'utilisa- 
tion de ce dialecte choisi dans l'administration, l'enseignement et les médias, ainsi qu'à la compilation et publication de matériels didactiques (dictionnaires, syllabaires, abécédaires, grammaires, journaux, etc.). Cette étude qui s'intitule The Standard Translation Dictionary as an Instrument in the Standardization of Fang n'apporte pas seulement des éléments de réponses à la standardisation du fan, elle fait également entrevoir aux linguistes et lexicographes travaillant sur d'autres langues gabonaises des pistes de recherche dans le domaine de la standardisation des langues.

Enfin, à côté de ces travaux publiés ou encore présentés et soutenus dans le cadre de mémoires ou de thèses, il faut souligner l'existence de recherches non datées. Il s'agit notamment des ouvrages intitulés Diccionario Pamue-Espanol, Vocabulary of the Fan Language in Western Africa (Osorio 1887), "Contribution à l'étude du folklore bantou: fables, devinettes et proverbes fang" (Tardy 1933) et Gramática pamue (Ndongo Esono 1956). Le travail d'Osorio, ajouté aux études susmentionnées, pourrait entrer dans la constitution d'une large base de données sur le fan. Le travail de Tardy, considérablement plus intéressant du point de la production de dictionnaires syntagmatiques, pourrait constituer le matériel de base pour la compilation et publication d'un dictionnaire des devinettes et proverbes en fan. De tels ouvrages de référence font encore défaut aux communautés linguistiques Fan.

En conclusion, tous les dictionnaires disponibles dans la langue fan sont des dictionnaires de traduction et centrés principalement sur le français. En outre, ils comportent un certain nombre de lacunes qui doivent être comblées. La plupart de ces travaux ne marquent pas les tons ainsi que la prononciation de la langue.

Grosso modo, ces lacunes s'appliquent également à l'unité-langue qui fait l'objet de la section suivante.

\section{Le Groupe Myene}

L'omyene est la langue maternelle de 5 pour cent de la population gabonaise. Les parlers de cette unité-langue sont principalement localisés dans trois provinces du Gabon: la province de l'Estuaire, le Moyen-Ogooué et l'Ogooué-Maritime. L'enenga est parlé dans le nord-est de Lambaréné, à proximité du lac Zilé. Le ghalwa se rencontre autour des lacs Onangué, Avanga, et Ezanga et sur l'île de Lambaréné. Le mpongwe est parlé à Libreville ainsi qu'à la Pointe Dénis. Enfin, le nkkomi est utilisé dans le sud-est de Port Gentil et dans les environs de la lagune du Fernand-Vaz.

Tous les travaux disponibles en littérature myene touchent aux domaines de la religion, l'ethnohistoire, la linguistique et la lexicographie. Les contributions religieuses incluent des traductions de la Bible, ainsi que des catéchismes, des recueils de cantiques et de prières (cf. Jacot 1894, Mission Catholique de Libreville 1894 et 1948). Ces travaux ont permis en outre de consolider l'œuvre d'évangélisation et de scolarisation (notamment grâce à la production de gram- 
maires, syllabaires et abécédaires dans les langues locales) des missionnaires catholiques et protestants.

Comme ce fut le cas pour le fan ci-dessus, il n'est pas sans intérêt de souligner que les syllabaires et abécédaires en question disponibles en langue omyene sont, en quelque sorte, des dictionnaires de prononciation. En effet, ils sont typiquement organisés de telle manière que les syllabogrammes (signes à valeur "phonétique") sont énumérés dans l'ordre vocalique a, i, u. Comparez les exemples suivants:

\begin{tabular}{|c|c|c|c|c|c|c|c|c|c|c|c|}
\hline & & $\mathbf{A}$ & $\mathbf{a}$ & & $A$ & $a$ & & & & & \\
\hline $\mathrm{a}$ & $\mathrm{a}$ & $\mathrm{a}$ & $\mathrm{a}$ & $\mathrm{a}$ & $\mathrm{a}$ & $\mathrm{a}$ & $\mathrm{a}$ & $\mathrm{a}$ & $\mathrm{a}$ & $\mathrm{a}$ & $\mathrm{a}$ \\
\hline \multirow[t]{2}{*}{$\mathrm{a}$} & $\mathrm{a}$ & $\mathrm{a}$ & $\mathrm{a}$ & $\mathrm{a}$ & $\mathrm{a}$ & $\mathrm{a}$ & $\mathrm{a}$ & $\mathrm{a}$ & $\mathrm{a}$ & $\mathrm{a}$ & $\mathrm{a}$ \\
\hline & & I & $\mathbf{i}$ & & $\mathscr{g}$ & $i$ & & & & & \\
\hline $\mathrm{i}$ & $\mathrm{i}$ & $\mathrm{i}$ & $\mathrm{i}$ & $\mathrm{i}$ & $\mathrm{i}$ & $\mathrm{i}$ & $\mathrm{i}$ & $\mathrm{i}$ & $\mathrm{i}$ & $\mathrm{i}$ & $\mathrm{i}$ \\
\hline \multirow[t]{2}{*}{$\mathrm{i}$} & $\mathrm{i}$ & $\mathrm{i}$ & $\mathrm{i}$ & $\mathrm{i}$ & $\mathrm{i}$ & $\mathrm{i}$ & $\mathrm{i}$ & $\mathrm{i}$ & $\mathrm{i}$ & $\mathrm{i}$ & $\mathrm{i}$ \\
\hline & & $\mathrm{U}$ & $\mathbf{u}$ & & $\mathscr{U}$ & $u$ & & & & & \\
\hline $\mathrm{u}$ & $\mathrm{u}$ & $\mathrm{u}$ & $\mathrm{u}$ & $\mathrm{u}$ & $\mathrm{u}$ & $\mathrm{u}$ & $\mathrm{u}$ & $\mathrm{u}$ & $\mathrm{u}$ & $\mathrm{u}$ & $\mathrm{u}$ \\
\hline $\mathrm{i}$ & $\mathrm{a}$ & $\mathrm{a}$ & $\mathrm{u}$ & $\mathrm{a}$ & $\mathrm{i}$ & $\mathrm{u}$ & $\mathrm{i}$ & $\mathrm{a}$ & $\mathrm{u}$ & $\mathrm{a}$ & $\mathrm{i}$ \\
\hline \multirow[t]{2}{*}{$\mathrm{u}$} & $\mathrm{i}$ & $\mathrm{a}$ & $\mathrm{i}$ & $\mathrm{u}$ & $\mathrm{a}$ & $\mathrm{u}$ & $\mathrm{i}$ & $\mathrm{a}$ & $\mathrm{u}$ & & \\
\hline & $\mathbf{a}$ & $\mathbf{i}$ & $\mathbf{u}$ & & $A$ & $\mathscr{U}$ & $\mathscr{g}$ & & & & \\
\hline
\end{tabular}

Figure 2: Quelques syllabogrammes du ghalwa (extrait du manuel Ezangô elônga zi galwa 1920, cité dans Mayer 1990: 83)

Sur le plan ethnohistorique, un certain nombre de travaux d'ensemble abordent entre autres les questions liées à l'histoire des migrations, à l'organisation sociale et à la description physique du milieu dans lequel vivent les populations $\underline{\text { Ngwe }}$-Myene (la mère Myene $\underline{e}$ ). Citons par exemple Haug (1903), Hauser (1954) et Raponda-Walker (1961). À côté de ces travaux de la première heure, figurent des travaux un peu plus récents, comme Ambouroue-Avaro (1981) et RatangaAtoz (1999). Le travail d'ensemble réalisé par Haug découle d'une observation minutieuse des peuples du Bas-Ogooué et de leurs mœurs. La contribution de Hauser constitue une étude de sociologie sur les Omyene du Bas-Gabon. Enfin le travail de Raponda-Walker publié avec une introduction et des cartes de M. Soret contient des documents indispensables, non seulement sur l'histoire des $\underline{\text { Ngwe }}$-Myene mais également sur d'autres peuples du Gabon. À côté de ces travaux d'ensemble, il y a des études limitées à certains groupes ethnolinguistiques de l'entité Myene. Citons par exemple les travaux de Gautier (1950, sur les Mpongwe)), Buléon (1887, 1892, sur les Nkomi), Agondjo-Okawe (1967, traité 
majeur de droit coutumier chez les Nkomi) et Gaulme (1976 et 1981, sur le royaume Nㅡomi).

Les travaux linguistiques en omyene sont exogènes. Les travaux de Delorme (1877) et de Raponda-Walker (1930-1934, 1961, 1998) sont certes des travaux lexicographiques mais ils sont précédés d'une description grammaticale de l'omyene. Quant aux travaux de description linguistique strictu sensu, on peut citer entre autres les études de Jacquot $(1978,1983)$, Mouguiama-Daouda (1991) et Kane (1998). Jacquot analyse le système phonologique et morphologique de l'omyenene. Plus tard, il réalise une étude d'ensemble sur les classes nominales dans les langues bantoues des groupes B10 (où figurent les six dialectes de l'omyene), B20 et B30. Mouguiama-Daouda se focalise pour sa part sur les niveaux d'analyse phonologique et morphologique du dialecte mpongwe. Enfin, Kane aborde également la variante mpongwe mais ici sous l'angle de la comparaison avec le français, langue officielle. Cette étude contrastive sur le mpongwe et le français réalisée par Kane a été précédée par aussi bien des descriptions synchroniques que diachroniques sur l'omyene. On peut citer Tchen-Damas (1990) et Gregoire (1990) sur la phonologie du mpongwe et du nkomi respectivement. Quant aux études diachroniques, la contribution de Rekanga (1986) sur l'omyene-nkomi figure en bonne place. Tous ces travaux linguistiques sont une mine d'informations pour tout lexicographe qui s'intéresserait à l'omyene.

La première publication omyene qui porte le titre de dictionnaire est l'ouvrage intitulé Dictionnaire français-mpongzwe par le R.P. Amable Delorme (1877). Ce dictionnaire qui couvre 354 pages, a été publié sous les auspices des Missionnaires de la Congrégation du Saint-Esprit et du Saint Cœur de Marie. Les lemmes sont présentés en français et accompagnés d'une traduction en mpongwe ainsi que de quelques exemples. L'ouvrage de Delorme est suivi quelques années plus tard de la publication du Dictionnaire mpongzè̀-français de Gachon (1881). L'ouvrage couvre 287 pages et se compose de deux sections. La première présente la grammaire du mpongwe, tandis que la deuxième contient le dictionnaire à proprement parler. Les lemmes sont classés par ordre alphabétique et selon la tradition du mot. ${ }^{2}$ Le dictionnaire a été une grande contribution à la traduction par Gachon de la Bible gnango ine agamba mi re tendo pa gou'ejango j'agnambie ji felio né TESTAMAN NOUNGOU NI TESTAMAN GNONA gou'inongo gni mpongoue publiée en 1891.

Le premier ouvrage majeur avec l'omyene et le français ne voit le jour qu'en 1934. Il s'agit du Dictionnaire mpongzé-français bilingue de l'Abbé Raponda-Walker (1930-1934) qui paraît chez les Presses de la Libre Lorraine à Metz. Le même auteur achève quelques années plus tard un ouvrage dans le sens inverse portant le titre Dictionnaire français-mpongwé (en abrégé DFM dans le reste de l'article) publié par l'Imprimerie Saint Paul à Brazzaville. Cette version du dictionnaire a été rééditée depuis sous les auspices de la Fondation Raponda Walker en 1995. La dernière version du travail comporte environ 8000 articles classés par ordre alphabétique selon la tradition du mot. La première par- 
tie de l'ouvrage est le dictionnaire à proprement parler, tandis que les posttextes ou deuxième partie du dictionnaire contiennent un exposé de la grammaire du mpongwe. Les deux volumes du dictionnaire (celui de 1930-1934 et celui de 1961) constituent indéniablement un dictionnaire complet. Ils se distinguent de leurs prédécesseurs par une élaboration soignée et par une grande richesse des mots-entrées ou lemmes. Dans la macrostructure, les substantifs prédominent, mais les autres classes de mots n'en sont pas pour autant négligées.

\begin{tabular}{|c|c|}
\hline Communiquer & $\begin{array}{l}\text { V. A. (transmettre) nóza; pa. Communiquer une } \\
\text { lettre, une maladie, nóz'ezango; nóze nkani. } \\
\text { Communiquer un ordre, bol'okenge. (Correspon- } \\
\text { dre). Communiquer par lettre, tendane yango; } \\
\text { une nouvelle, myeze ntsango. (Aboutir) punda, } \\
\text { pókósa. Le Fernand-Vaz communique avec } \\
\text { l'Océan, Eliwe-Nkómi zi punda go ntsuwa. Ce } \\
\text { chemin communique avec la plaine, mpónó yinó } \\
\text { yi pókósa g'orove. Se communiquer, nógana. Le } \\
\text { pian se communique, abukwè mi nógana. }\end{array}$ \\
\hline
\end{tabular}

Exemple textuel 1: Article communiquer (extrait de DFM 1995: 119)

En résumé, dans cet état des lieux sur la littérature myenene, il apparaît clairement que le dialecte le plus abondamment documenté est le mpongwe. La raison la plus évidente de cet état de fait est la position géographique. En effet, situées sur le littoral, les populations Mpongwe furent les premières à entrer en contact avec les explorateurs européens. Le domaine que nous allons à présent examiner fournit une littérature moins riche que le précédant ainsi qu'une matière lexicographique quasi inexistante.

\section{Le Groupe Mekana-Menaa}

Les parlers qui constituent cette unité-langue sont disséminés dans six provinces du Gabon, à savoir: l'Estuaire, le Haut-Ogooué; le Moyen-Ogooué, la Ngounié, l'Ogooué-Lolo et l'Ogooué-Ivindo. L'akele est parlé à Lambaréné et Sindara, l'ungom au nord de Franceville, à Koulamoutou et aux environs de Mékambo. Le lisighu et le metombolo se rencontrent à Lastoursville, le seki à Cocobeach. Le shake est présent à Booué et Lastoursville, le tumbidi à Mbigou et Malinga. Le lendambomo est parlé à Booué, Okondja ainsi qu'au sud de Mékambo. Enfin, le wumpfu se rencontre à Mbigou, Malinga et au nord de Franceville.

L'essentiel des travaux sur le groupe mekana-menaa couvre les domaines de l'ethnohistoire et la linguistique. Parmi les travaux consacrés à l'ethnohistoire mentionnons ceux d'Avelot (1911) et Deschamps (1962). Dans le premier essai, l'auteur décrit avec minutie les pratiques religieuses chez les populations de l'ethnie Akele. Le second essai est un ouvrage de référence en ethnohistoire. 
En effet, il contient les traditions orales recueillies sur l'ensemble des ethnies du Gabon. D'une manière générale, les migrations des ethnies de cette unité-langue ne sont pas très bien connues. Toutefois, pour les peuples Ndambomo, Wumpfu et Shake entre autres, les récits oraux mentionnent avec insistance deux faits, à savoir: la guerre de pupu et la querelle des ancêtres à propos d'une antilope prise au filet au cours d'une partie de chasse en groupe. Ces faits sont à l'origine de la dispersion des ethnies précitées.

En proie également à la dispersion suite à la même guerre de pupu, le peuple Akele venu du Nord-Est va essaimer les provinces du Haut-Ogooué (l'ethnie Mbanwe $)$, la Ngounié (les ethnies Tumbidi et Metombolo) et l'OgoouéLolo (les ethnies Sighu et Bungom). Dans leur migration, les Bakele croisent le chemin des Beseki. S'engage alors une épreuve de force qui va contraindre les Beseki à se scinder en quatre groupes qui occuperont respectivement la Noya jusqu'à l'Estuaire de la Mondah et ses affluents, Libreville, la zone littorale du Como et l'Ogooué.

L'ensemble des travaux sur les parlers du groupe mekana-menaa s'inscrivent dans la perspective de la linguistique synchronique et inclut la contribution des auteurs suivants: Jacquot (1983), Ondo-Mebiame (1989) et Manfoumbi (1989). L'ouvrage de Jacquot (qui s'inscrit dans la longue lignée des travaux de classification sur les langues Bantu) examine le système des classes nominales dans les langues Bantu des groupes B10, B20, et B30. Pour chacune des langues retenues dans ce groupe, l'auteur propose un tableau des classificateurs dans lequel les classes s'associent éventuellement par paires en opposition singulierpluriel pour former des genres. Les nombreux commentaires qui sous-tendent ces travaux font de cet ouvrage un outil morphologique indispensable à tout chercheur intéressé par les parlers de l'unité-langue mekana-menaa qui appartiennent majoritairement au groupe B20 de Guthrie. Il n'est pas inutile de signaler que ce travail à fait l'objet d'une critique dans Blanchon (1988).

Les deux dernières études se penchent respectivement sur la phonologie et la morphologie du seki et du shake. La première partie du travail d'OndoMebiame est consacrée à la phonologie paradigmatique (tonèmes, voyelles et consonnes) et à la phonologie syntagmatique (étude des structures syllabiques). Dans la seconde partie de l'essai, le descripteur se propose de prouver l'appartenance du seki au domaine Bantu. Pour y arriver, il sollicite les critères élaborés par Guthrie (1948). Cette partie s'achève sur la présentation du système nominal du seki qui compte douze classes pour onze genres. On observera que la démarche de l'auteur épouse dans ses grandes lignes les principes développés par les linguistes du cercle de Prague. Avec cependant une préférence accordée au système théorique du fonctionnalisme martinétien.

Manfoumbi épouse la même démarche classique: au niveau d'analyse phonologique, l'auteur procède à l'identification des phonèmes du shake en recourant notamment au jeu des paires minimales et à la commutation. La composante descriptive morphologique aborde pour sa part les classes nominales du shake. In fine, mentionnons que ces deux mémoires de maîtrise ont 
fait l'objet de publication d'articles, respectivement Ondo-Mebiame et NzangBié (1990) et Manfoumbi et Zambibonda (1990). L'ensemble de ces travaux linguistiques sont indispensables à la rédaction de la partie introductive du dictionnaire, parce que c'est cette dernière qui est censée présenter entre autres les informations phonologiques et morphologiques des parlers qui font l'objet de production dictionnairique. Cependant, force est de constater qu'il n'existe pas dans cette unité-langue de matière lexicographique stricto sensu. Qu'à cela ne tienne, la production d'une telle littérature n'est pas impossible à partir de ce qui existe déjà. À titre d'illustration, les travaux ethnohistoriques dans ce groupe pourraient après synthèse entrer en ligne de compte dans la rédaction des aperçus historiques et les présentations ethnohistoriques des populations utilisant les langues du groupe dit mekana-menaa. Le regroupement, notamment, de toutes les dénominations des lieux d'importance historique, les noms des chefs les plus importants dans chaque groupe ethnolinguistique, etc. pourraient ainsi constituer le matériau de base des documents annexes susceptibles d'apparaître dans les posttextes des ouvrages de référence lexicographique. Par rapport à ce point, les dictionnaires et les encyclopédies doivent être considérés comme des véhicules de types textuels, c'est-à-dire que les dictionnaires renferment une pluralité de textes d'origine diverse. Les prétextes (ensemble des textes présentés avant la section $\mathrm{A}-\mathrm{Z}$ ou nomenclature) abritent généralement le guide pour les utilisateurs ainsi que la mini grammaire de la langue traitée; par contre, les posttextes (ensemble des textes figurant après la section $\mathrm{A}-\mathrm{Z}$ ou nomenclature) offrent généralement aux lexicographes une plateforme pour la présentation des proverbes, devinettes, anthroponymes, toponymes, etc. Ce sont là des possibilités que ne devraient pas sous-estimer les lexicographes travaillant sur les langues du groupe mekana-menaa en particulier. L'unitélangue qui va suivre présente également une matière lexicographique relativement pauvre.

\section{Le Groupe Mekona-Mangọte}

Les parlers qui composent cette unité-langue sont éparpillés dans cinq des neuf provinces que compte le Gabon, à savoir: l'Estuaire, le Haut-Ogooué, la Ngounié, l'Ogooué-Lolo et l'Ogooué-Ivindo. L'ikota est parlé à Booué et Makokou, le benga est signalé au nord de Libreville au Cap Estérias et à la Pointe SantaClara. Le shamayi se rencontre à Makokou et Okondja, le mahongwe et le bakola à Makokou. Enfin, le ndasha est parlé à Mbigou, Mandjaye et Okondja (les Bandasha sont également signalés en République du Congo-Brazzaville, plus précisément à Mossendjo et à Dolosie dans la région du Nyari, dans la commune de Moutamba).

L'essentiel de la littérature mekona-mangọte couvre les domaines de la religion, l'ethnohistoire et la linguistique. Du point de vue religieux, mentionnons les contributions de Biton (1903) et Lamour (1936). Les ouvrages de ces deux auteurs ont une caractéristique commune: ce sont des livres de questions 
ou catéchismes sur l'idéologie catholique en langue ikota. Ce qui distingue ces deux ouvrages est évidemment le fait que le premier est publié tandis que le second nous est parvenu uniquement sous sa forme manuscrite.

Nombre d'ouvrages ethnohistoriques existent dans ce groupe. Mentionnons notamment le travail de Even (1937) sur les ethnies Obamba et Ndasha. Pour l'objet qui nous occupe, nous nous intéresserons à l'ethnie Ndasha. Un des chefs historiques de ce groupe, Oloupi, servit d'informateur à l'auteur, à l'époque où ce dernier occupait les fonctions d'administrateur adjoint aux colonies. Dans cet ouvrage, l'auteur décrit avec diligence les rites de passage dans les sociétés initiatiques suivantes: Mungala, Ngoyi, et Lishimbu. En outre, nous trouvons le récit de la migration des ethnies de ce groupe chez Deschamps (1962). Au chapitre 7 de son ouvrage, l'auteur mentionne deux faits récurrents dans les traditions orales: la guerre de pupu et la querelle des ancêtres à propos d'une antilope prise au filet. Ces faits sont à l'origine de la dispersion des ethnies précitées.

Le pays d'origine des Benga se situerait aux confins du Cameroun, de Centrafrique et du Congo. Sous la pression des Bekwel et des Bafan, les Benga ainsi que les Kota se dirigèrent vers le Gabon. C'est dans le Haut-Ivindo que les deux peuples se séparèrent à la suite de la guerre de pupu. Après cet épisode guerrier, les Benga se dirigèrent en ordre dispersée vers le Cameroun, la Guinée-Équatoriale et le Cap Estérias ainsi que la Pointe Santa-Clara (qui constituent avec Cocobeach les principaux foyers de concentration des Benga au Gabon).

$\mathrm{Du}$ point de vue linguistique, les parlers benga et ikota apparaissent comme les plus connus et les plus abondamment documentés. Pour ce qui est du benga, mentionnons les contributions des auteurs suivants: Mackey (1855), Meinhof (1889-1890), Perez et Sorinas (1928) et Essono (1988). Il convient de s'arrêter sur le dernier auteur dont le travail s'inscrit dans le fonctionnalisme. Ce travail comporte deux parties: la phonologie et la morphologie. Dans la première composante descriptive, l'auteur procède à l'identification des phonèmes du benga. Dans le second niveau d'analyse, le descripteur établit d'abord l'appartenance du benga au domaine Bantu en s'appuyant sur les critères élaborés par Guthrie (1948). Ensuite, l'auteur s'attèle à l'examen des classes nominales. L'analyse établit vingt-quatre classes nominales qui s'associent par paires en opposition singulier-pluriel pour former huit genres. Ce travail a fait l'objet de la publication d'un article "Bønga" (1990) par le même auteur dans la Revue Gabonaise des Sciences de l'Homme. S'agissant de l'ikota, l'article d'Hombert (1990) porte sur la phonologie paradigmatique (systèmes vocalique et consonantique) de ce parler. Cet article s'achève sur un inventaire des symboles retenus pour l'Alphabet Scientifique du Gabon (ASG) ainsi que quelques proverbes. Pour l'ikota, il faut mentionner le travail de Gamille (1997) dont l'intérêt est beaucoup plus sociolinguistique que linguistique stricto sensu. Ce travail est abordé avec une arrière-pensée comparative: l'auteur examine un ensemble de textes kota du Gabon et du Congo sous l'angle de la variation. In fine, men- 
tionnons les contributions des auteurs suivants: Sima Mvé (1996) et Mavoungou (1998) sur respectivement le mahongwe et le ndasha. Sur un plan morphologique, l'ouvrage de Jacquot (1978) que nous citons plus haut a valeur de source. Le seul ouvrage à caractère lexicographique dont nous disposons sur ce groupe est du R.P. Perron (1964). Il s'agit d'un lexique bilingue français-ikota publié en deux volumes sur l'initiative de la Mission Catholique de Makokou. Comme informations microstructurelles, ce bilingue est une reprise des notes manuscrites du R.P. Lamour. Comme ce fut le cas pour les autres unités-langues, les données disponibles sur les plans ethnohistorique et linguistique notamment constituent une matière dictionnairique non négligeable. Les travaux lexicographiques en cours au Gabon gagneraient à inclure les langues du groupe dit mekona-mangote. L'unité-langue qui va suivre présente une matière lexicographique relativement plus étoffée.

\section{Le Groupe Membe}

Les parlers de ce groupe se retrouvent principalement dans trois des neuf provinces du Gabon, à savoir: la Ngounié, la Nyanga, l'Ogooué-Ivindo et l'Ogooué-Lolo. Le ghetsogho est parlé à Mouila, Moabi et Sindara. Le ghepinzi et le ghevhiya sont localisés à Mouila et le ghevhovhe à Koulamoutou. Le ghehimbaka, l'ebongwe et le kota-kota se rencontrent principalement à Booué et à Mimongo.

Tous les travaux disponibles en littérature membe touchent aux domaines de la linguistique et de la religion. Ces travaux, qui contiennent beaucoup d'informations concernant le lexique ainsi que la grammaire des parlers de ce groupe, peuvent être employés comme point de départ pour la production de nouveaux dictionnaires. Nombre de travaux linguistiques portant sur les parlers de ce groupe ont été présentés et soutenus à l'Université Omar Bongo ou faits par des étudiants (gabonais ou non) dans les universités d'Europe. Mentionnons les contributions de Marchal-Nasse (1979 et 1990, sur le ghetsogho), Idiata (1989, sur le ghepinzi), Blanchon (1988, sur le ghevhiya) et Van der Veen (1991, 1992, 1999, sur le groupe B30 et le ghevhiya).

Les études qui ont spécifiquement traité des questions lexicographiques dans cette unité-langue incluent des travaux des auteurs suivants: RapondaWalker (n.d.) et Bodinga-bwa-Bodinga et Van der Veen (1990). La contribution de Raponda-Walker est un dictionnaire bilingue intitulé Dictionnaire getsogofrançais. L'ouvrage contient plus de 5000 articles couvrant 237 pages. Des lemmes en ghetsogho sont proposés avec des traductions en français ainsi que quelques exemples. L'auteur a également produit une édition du dictionnaire dans le sens inverse avec le français comme langue source et le ghetsogho comme langue cible. L'article de Bodinga-bwa-Bodinga et Van der Veen "Plantes utiles des Evia" traite des noms de plantes ainsi que de leurs propriétés médicinales. Cette étude rejoint dans ses grandes lignes l'ouvrage Les plantes utiles du Gabon publié en 1961 par Raponda-Walker et Sillans et contient envi- 
ron 608 items lexicaux en ghevhiya. Les entrées du lexique apparaissent en trois colonnes. Les noms scientifiques des plantes sont présentés dans la première colonne dans l'ordre alphabétique. Les noms en ghevhiya apparaissent dans la deuxième colonne. La dernière colonne traite des propriétés thérapeutiques des plantes énumérées. Ceci est illustré par l'exemple suivant:

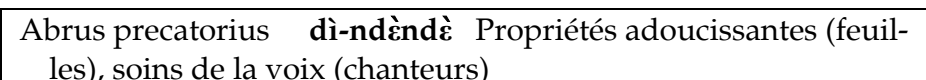

Exemple textuel 2: Article Abrus precatorius (extrait de Bodinga-bwa-Bodinga et Van der Veen 1990: 30)

Enfin, la collaboration de Van der Veen et Bodinga-bwa-Bodinga inclut également la production d'un dictionnaire bilingue: Gedandedi sa Geviya/Dictionnaire Geviya-Français (2002, en abrégé DGF dans le reste de l'article) paru aux Éditions Peeters. Ce dictionnaire, fruit d'une longue collaboration scientifique fructueuse entre Van der Veen et Bodinga-bwa-Bodinga, marque le début d'une nouvelle ère dans la compilation des dictionnaires au Gabon (Mavoungou 2004). La macrostructure du DGF contient environ 6200 articles classés par ordre alphabétique sous la lettre initiale du thème de chaque lemme. Les unités de traitement sont précédées de leurs préfixes respectifs entre parenthèses d'une part, et sont d'autre part suivies d'une indication de leurs schèmes tonals respectifs accompagnée d'une spécification de la catégorie grammaticale (partie du discours) ainsi que du numéro de classe auxquels appartiennent les lemmes. Considérons l'exemple suivant:

(mo-)gadi H n 1/2 • 1épouse. Tsikà mógádì ámé wá mósòngá nà lélé. Laisse ma première épouse tranquille. Mògàdì á ngà. Femme d'autrui. Voir mo-geto. • $2^{\circ}$ (fig.) femelle (d'un animal) Mògàdì á ngòèà. La femelle du potamochère. Mògàdì á èd ’̀mbc̀. Brébis. Mògàdì á gèvàlàngò. Cane.

Exemple textuel 3: Article (mo-)gadi (extrait de DGF 2002: 136-137)

Après la parution du DGF, le ghevhiya, compte tenu de la sérieuse menace de sa disparition ${ }^{3}$, pourrait s'enrichir avec la publication d'autres ouvrages de référence lexicographique notamment les dictionnaires syntagmatiques, c'est-à-dire les dictionnaires des proverbes et devinettes. À ces dictionnaires, on pourrait également ajouter des dictionnaires de spécialité dit dictionnaires LSP couvrant notamment le domaine de la pharmacopée traditionnelle (mais ce n'est pas là l'objet de notre discussion). Les publications suivantes pourraient constituer la matière lexicographique de tous ces dictionnaires: Bodinga-bwa-Bodinga (1969) et Bodinga-bwa-Bodinga et Van der Veen (1993, 1995). Après la parution du DGF, l'autre travail de référence lexicographique pour ce groupe est la contribution de Mickala Manfoumbi (2004) qui s'intitule Lexique pove-français/français-pove (en abrégé LPFFP dans le reste de l'article). Ce lexique présente une 
nomenclature d'environ 8227 mots répartis sur 725 pages. Cette nomenclature déploie ses données en quatre colonnes. Dans la section pove-français en particulier, la première colonne identifie les lemmes (classés par ordre alphabétique sous la lettre initiale du thème de chaque mot) ainsi que les éléments de formation (affixes et formants savants) de même que les variantes des mots-entrées ou vedettes. La deuxième colonne donne les indices ou numéros de classe. La troisième présente la forme entière des lemmes selon l'opposition singulierpluriel et la quatrième colonne donne la traduction en français des lemmes proposés. L'auteur simplifie ses données dans le souci majeur de fournir un manuel de première initiation. Il y a donc chez lui un souci constant d'adapter l'ouvrage au public. Le lecteur aura une idée du type de répertoire dont il s'agit d'après le bref fragment suivant:

\begin{tabular}{|c|c|c|c|}
\hline + sòlà & 3,4 & musola & "purée, bouillie" \\
\hline + sòlè-sòlè & 7,8 & $\begin{array}{l}\text { misola } \\
\text { gesolesole } \\
\text { bisolesole }\end{array}$ & "instigateur" \\
\hline + sòlò & 5,6 & $\begin{array}{l}\text { esolo } \\
\text { masolo }\end{array}$ & "détection, flair" \\
\hline
\end{tabular}

Exemple textuel 4: Articles + sòlà, + sòlè-sòlè et + sòlò (extrait du LPFFP 2004: 312)

En revanche dans la section français-pove, les items lexicaux en français et en italique apparaissent dans la première colonne. La deuxième colonne présente leurs équivalents pove en gras et sous la forme du thème. Les indices de classe apparaissent dans la troisième colonne et les items lexicaux correspondant aux thèmes en pove apparaissent dans la dernière colonne sous leur forme complète. La typographie est soignée, claire et aérée et elle facilite la lisibilité. Ce lexique qui frappe par sa richesse et sa variété est le premier ouvrage de référence sur le pove. C'est dire tout le symbole qu'il représente pour la communauté Pove toute entière. L'unité-langue qui va suivre présente une matière lexicographique un peu plus importante.

\section{Le Groupe Merye}

Les parlers qui constituent cette unité-langue se rencontrent dans quatre des neuf provinces que compte le pays, à savoir: la Ngounié, la Nyanga, l'OgoouéMaritime et l'Ogooué-Lolo. Le ghisira est parlé dans les localités de Fougamou, Mandji et Ndougou, le ghivharama à Setté-Cama et Gamba. Le ghivhungu est signalé à Mandji, Yetsou et Moabi. Le yipunu est parlé à Mouila, Tchibanga, Ndendé, Mabanda et Moabi. Le yilumbu est parlé le long de l'axe routier Tchibanga-Mayumba ainsi qu'à Gamba et Setté-Cama. Le yisangu se rencontre dans les localités de Mimongo, Mbigou, Koulamoutou jusqu'à Iboundji. Le ngubi est 
parlé dans la lagune Iguéla, le civili à Mayumba et Ndindi et le yirimba à Moabi. Enfin, le yighama est parlé entre Mayumba et Tchibanga. Signalons en outre que quelques parlers de ce groupe se rencontrent également dans quelques pays voisins du Gabon. C'est le cas du yipunu et du yilumbu que l'on rencontre au Congo-Brazzaville et au Congo-Kinshasa d'une part; d'autre part, l'on trouve également le civili au Congo-Brazzaville (Pointe Noire) et dans l'enclave du Cabinda.

De façon générale, la littérature merye couvre les domaines de la religion, l'ethnohistoire, la littérature orale et la linguistique. Parmi les auteurs de productions religieuses, citons Garnier $(1897,1903,1904 ; 1900)$ et Murard (1903, 1903a). Le premier est l'auteur de quatre essais consacrés au yilumbu (variante dialectale de la Nyanga, lagune Banio) et au civili, à savoir: Katesisa $i$ gheghe nesi ma loghi ma dzambi mu mbembo i-lumbu (1897), Мu mbembo kivili ki mayumbe (1903), M'ambu ma nzambi mo make mu katesisa (1904) et Syllabaire i-lumbu keti miganda mio mi teti mi u ranganga mu mbembo i-lumbu (1900). Les trois premiers ouvrages ressortissent de la liturgie tandis que le dernier constitue un exercice scolaire. Le second, à la différence du premier, est l'auteur d'un ouvrage en deux volumes consacré au yilumbu parlé dans la province de l'Ogooué-Maritime (cantons de Ndugu-Lagune et Basse-Nyanga). Il s'agit de Katsisu i neni i rendilu mu mbembo bis'Sette-Cama (1903), et Katsisu i keki i rendilu mu mbembo bis'Sette-Cama (1903a). Mentionnons également pour yilumba le travail Catéchisme de la doctrine chrétienne = Katsisu mu mbembo hi lumba hi bis'sette-cama (Mission Catholique de Setté-Cama 1933). Les autres publications à caractère biblique et liturgique sont consacrées aux parlers yipunu et ghisira. Nous avons respectivement les ouvrages: Cantiques $=$ Gnimbu tsi niambi (1903b) ainsi que Catechisme gisira (1924). Enfin, pour ce dernier ouvrage consacré au ghisira mentionnons également les contributions de Buléon (1899) sur ce même parler. L'ensemble de ces travaux qui vont du genre monolingue aux productions interdialectales constitue une matière lexicographique non négligeable.

Nombre de travaux linguistiques existent dans cette unité-langue. Citons par exemple les contributions des auteurs suivants sur le ghisira, le yipunu, le yisangu et le civili respectivement: Koumba (1990), Mouguiama-Daouda (1991) et Dodo-Bounguedza (1992); Nsuka-Nkutsi (1980) et Mickala-Manfoumbi (1986); Ondo-Mebiame (1989), Idiata (1993, 1998) et Mickala-Manfoumbi (1989); Ndamba (1977), Mabika Mbokou (1999) et Ndinga-Koumba-Binza (2000).

Les travaux lexicographiques à proprement parler dans cette unité-langue débutent avec la publication du bilingue Dictionnaire vili-français par le R.P. Marichelle (1902). Ce dictionnaire, qui couvre 224 pages, a été publié à Loango. Dans la préface, l'auteur précise que la parution du Dictionnaire vili-français vient compléter l'œuvre du R.P. Derouet (n.d.), auteur du Dictionnaire françaisvili. L'ouvrage de Marichelle comporte un bref aperçu du parler civili. Les articles du dictionnaire fournissent les données lexicographiques suivantes: une unité de traitement en civili, une traduction en français ainsi que quelques exemples. Comparez l'exemple suivant: 
Dasuka, vn. P. isi, - Se mettre en colère, se fâcher, s'irriter, se monter. I be n'-kamba, u a dusuka to, je lui ai dit la chose, mais il ne fait que se fâcher. Lemboa $k u-$, ne te fâche pas.

Exemple textuel 5: Article Dasuka (extrait de Marichelle 1902: 46)

Mis à part les travaux lexicographiques sur le civili, on note également quelques productions à caractère dictionnairique en yisangu, yipunu et en ghisira. Naidaillac (1992, 1992a) en collaboration avec l'équipe de l'ALGAB ${ }^{4}$ a produit un travail lexicographique intitulé Lexique isangu-français. Idiata (1993) aborde également quelques aspects des lexiques spécialisés en yisangu. Quant au yipunu, il existe une littérature lexicographique pionnière réalisée par exemple par le R.P. Bonneau (1956) ou encore par le Christian Missionary Alliance (CMA) sous les auspices de l'Église Evangélique du Sud-Gabon (1966). En dehors de ces travaux de la première heure, il y a un ensemble de travaux lexicologiques et lexicographiques relativement plus récents. Citons par exemple RittaudHutinet (1980) et Kwenzi-Mikala $(1989,1990)$. Pour ce dernier auteur, il faut souligner l'existence d'un ensemble de travaux sous presse sur notamment des questions d'anthroponymie (Kwenzi-Mikala 1990a) et des lexiques spécialisés (qui ne seront pas abordés ici). Enfin, pour le yipunu citons un travail lexicographique sous forme électronique, il s'agit Dictionnaire informatisé du pounou par Blanchon (cité dans Blanchon 1997a). Ce dictionnaire compte pour le moment 4272 lemmes, accompagnés de leurs étymons.

Pour le ghisira, l'ouvrage lexicographique sur lequel il convient de s'arrêter s'intitule Petit dictionnaire français-gisira-ngosi et éléments de verbes rédigé par une religieuse de la congrégation de l'Immaculé Conception: la sœur Lemoine (1994). Comme son titre l'indique, cet ouvrage est un lexique présentant une nomenclature de 2619 lemmes. À la suite du lemme ou unité de traitement (en français et en gras), le traitement microstructurel présente les données suivantes: la traduction en ghisira du lemme français (il s'agit précisément de l'unité lexicale ghisira au singulier suivie du morphème du pluriel) et éventuellement d'autres unités lexicales en français (en italique) appartenant au même champ sémantique que le lemme avec leurs équivalent en ghisira. Comparez l'exemple suivant:

$\begin{aligned} \text { mouton: } & \text { didombi-ma } \\ & \text { bélier: didombi di mulumi } \\ & \text { brebis: mwana didombi- } \\ & \text { dindombi di mugatsi }\end{aligned}$

Exemple textuel 6: Article mouton (extrait de Lemoine 1994: 74)

Par rapport aux sources primaires du lexique, dans les notes introductives de l'ouvrage il est clairement stipulé que ce travail repose sur des exemples d'oc- 
currences orales du ghisira, qui ont été collectées sur plusieurs années (sœur Lemoine a exercée pendant 24 ans à la Mission Sainte-Croix de Mandji-Ndolou) à partir de diverses sources (citons notamment les 600 lectures et évangiles des trois années liturgiques traduits par l'auteur pour le compte de la communauté Ghisira de Mandji-Ndolou). L'orthographe utilisée dans cette production à caractère dictionnairique repose largement sur l'ensemble des graphies du français.

Les items lexicaux de la nomenclature présentent des lacunes à la fois sur le plan segmental et suprasegmental. Pour tous les travaux précurseurs, réserves (voir Nyangone Assam et Mavoungou 2000 et Mavoungou 2001, 2001a, 2002) ont déjà été faites en ce qui concerne l'absence de tons dans les transcriptions écrites des mots (a priori sans indications tonales, on ne peut pas s'attendre à ce qu'un usager reproduise correctement le système phonétique des langues décrites). S'agissant des voyelles en particulier, avec le système orthographique du français comme point de référence, Lemoine essaie de distinguer en ghisira les voyelles qui sont phonologiquement distinctives à l'aide notamment de signes diacritiques (signes graphiques qui permettent de modifier la valeur de base d'un graphème). Par exemple, $[\varepsilon]$ et [o] sont toutes les deux représentées par è et $\ddot{o}$.

Considérablement plus intéressant du point de vue métalexicographique, le lexique de Lemoine présente un cadre structurel. En effet, la nomenclature est encadrée des prétextes et des posttextes. La grande richesse de l'ouvrage de Lemoine réside dans la présentation d'une variété de types textuels comme posttextes. Le texte central au niveau des posttextes sur lequel il convient de s'arrêter est intitulé VERBES. C'est dans ce texte que nous trouvons la conjugaison de certains verbes (entre autres, être, voir, aimer, manger, boire, entendre à l'indicatif présent, à l'imparfait, au passé, au futur, à l'impératif, au subjonctif, etc.) en ghisira. À la périphérie de ce texte central gravitent un ensemble d'autres textes et non des moindres. Le premier texte qu'il convient de mentionner s'intitule MONNAIE. Comparez l'illustration suivante:

\begin{tabular}{|r|l|}
\hline $5 \mathrm{~F}$ & doli mosi \\
\hline $10 \mathrm{~F}$ & doli bèi \\
\hline $15 \mathrm{~F}$ & doli irèru \\
\hline $20 \mathrm{~F}$ & doli ina \\
\hline $25 \mathrm{~F}$ & doli iranu \\
\hline $30 \mathrm{~F}$ & doli siamanu \\
\hline $35 \mathrm{~F}$ & doli ina gu rèru \\
\hline $40 \mathrm{~F}$ & doli gimana \\
\hline $45 \mathrm{~F}$ & doli kambumosi \\
\hline $50 \mathrm{~F}$ & digumi di doli \\
\hline$\ldots$ & $\ldots$ \\
\hline
\end{tabular}

Exemple textuel 7: Posttexte MONNAIE (extrait de Lemoine 1994: 115) 
Le tableau ci-dessus présente une liste des dénominations allant de 5F CFA à 200000 F CFA. Cette liste pourrait être utile pour la réception et la production des textes aussi bien pour les locuteurs natifs que pour les apprenants du ghisira.

Le second posttexte qu'il convient de souligner est intitulé DANSES. Considérons l'exemple suivant:

\begin{tabular}{|ll|}
\hline Bwiti: & $\begin{array}{l}\text { Pour trouver le coupable } \\
\text { Initiation avec iboga: ama gwingène gu mbwiti: il rentre } \\
\text { dans le bwiti avec l'iboga } \\
\text { Mwiri: }\end{array}$ \\
Nyemba: & Pour un garçon devenu homme, fort, courageaux \\
Mugulu: & Pour une fille devenue femme \\
Mabanzi: & Pour se soigner avec la musique: Disigi \\
Girina: & Pour avoir des enfants, chasser les esprits: avec l'iboga \\
Bilumbu: & Pourquoi enfants morts avec l'iboga \\
Mavassa: & Avec les esprits avec l'iboga \\
Divanda-mavanda: & Pour avoir des jumeaux \\
Mukwèlengi: & Mutu a ne mavanda: homme avec fétiches \\
Ntsimba: & (nom d'un oiseau): garçons et filles \\
\hline
\end{tabular}

Exemple textuel 8: Posttexte DANSES (extrait de Lemoine 1994: 129)

Dans l'exemple textuel ci-dessus, il est intéressant de noter que l'auteur ne se contente pas de présenter des traductions pour chaque unité lexicale ghisira. Le traitement inclut aussi bien les traductions que les données contextuelles (étiquettes) et les données cotextuelles (les exemples). L'accent dans cet extrait textuel est mis sur les items lexicaux ghisira ayant une forte charge sémanticoculturelle notamment les pratiques médico-magiques.

D'un point de vue graphique, il faut noter que le système orthographique $\mathrm{du}$ français a des signes pour la gémination ou redoublement consonantique, cependant ce n'est pas le cas des langues gabonaises et le ghisira en particulier. Sous l'influence de sa langue maternelle, Lemoine a écrit le $s$ du ghisira comme le français ss qui est prononcé [s], par exemple Mavassa (au lieu de Mavasa). Pour ce qui est de la division des mots, les langues africaines ont deux traditions d'écriture distincte, à savoir: une écriture conjonctive et une écriture disjonctive. Dans une écriture conjonctive, les mots présentent une structure interne complexe (par exemple les préfixes verbaux, les formatifs, les radicaux et leurs extensions formant un tout). Par contre, une écriture disjonctive présente une structure interne plus simple: les affixes (préfixes, infixes et suffixes) des unités lexicales apparaissent séparément. Lemoine a fait le choix d'une écriture disjonctive (cf. ama gwingène gu mbwiti). Ce choix est beaucoup plus visible dans le troisième et dernier posttexte de notre étude: PRIÈRES. Comparez l'exemple suivant: 


Taï étu ndya u gu yulu, dina dyagu di ki du misa, divinda
dyagu di ki ruga, tondini agu i ki vagu, gu tsi na gu
yulu. Du vègi ne muni bigui byètu bi tsufu tsyotsu, u ku
du kébila gu péchés, tyètù; nève dua kébili, bisi ba du
va mabi; u ya du sila gu fama gu péchés tsi dua sindi-
gitsa, tu du kani gu mabi. Amen

Exemple textuel 9: Posttexte PRIÈRES (extrait de Lemoine 1994: 131)

Cet exemple textuel ajouté notamment aux 600 lectures et évangiles des trois années liturgiques traduits du français au ghisira par Lemoine pourraient entrer dans la constitution d'un corpus électronique ghisira d'où l'on pourrait dériver un ensemble d'ouvrages lexicographiques (dictionnaires monolingues, bilingues ou multilingues). L'unité-langue qui va suivre présente comme le groupe merye un fonds dictionnairique et d'intéressantes perspectives de développement de la discipline lexicographique.

\section{Le Groupe Metye}

Les parlers de ce groupe sont principalement dispersés dans quatre des neuf provinces du Gabon, à savoir: le Haut-Ogooué, la Ngounié, l'Ogooué-Ivindo et l'Ogooué-Lolo.

Le yinzebi est le parler le plus largement répandu du groupe et il est localisé dans des régions telles que Mbigou, Lebamba, Malinga, Lastoursville, Koulamoutou, Pana-Iboundji et Mounana-Bakoumba. Le yitsengi est parlé à Lastoursville, à Moanda et à Bakoumba, et le yimwele à Mbigou. Le yivhili est parlé à Booué et à Sindara, et liwanzi à Lastoursville et à Moanda. Le liduma est parlé à Lastoursville et le yibongo à Moabi.

La littérature metye couvre essentiellement les domaines de la religion et de la linguistique. Il y a toutefois un certain nombre de travaux qui proviennent de l'ethnohistoire et de la littérature orale mais dont nous ne parlerons pas ici. Les publications à caractère biblique et liturgique sont essentiellement consacrées à liduma. Citons par exemple Catéchisme en langue adouma (Dahin 1891) et Bivovili bya bakretyeni ba baduma (Reeb 1895). Les travaux linguistiques incluent pour leur part les contributions des auteurs suivants sur liduma, liwanzi et le yibongo respectivement: Mouélé (1997), Dodo-Bounguendza (1986), et Raponda-Walker (1937). À côté des études sur liduma, liwanzi et le yibongo entre autres, il y a un certain nombre de travaux sur le yinzebi présentés et soutenus à l'Université Omar Bongo ou dans les universités européennes. Citons par exemple: Chela (1998), Makoungou (1999), Ndoumou Mba (1999) et MarchalNasse (1989). Pour cette dernière contribution, en 1990, le même auteur publie dans la Revue Gabonaise des Sciences de l'Homme l'article "Yinzzbi". Tous ces travaux aussi bien religieux que linguistiques dans les langues précitées constituent une matière lexicographique non négligeable. 
Le premier travail en lexicographie dans ce groupe avec le français et liduma est une contribution du R.P. Dahin intitulé Vocabulaire français-aduma (1893). Le même auteur achève quelques années plus tard l'édition dans le sens inverse du même ouvrage: Vocabulaire aduma-français (1895). Ces lexiques constituent certainement un ouvrage complet en deux volumes. Les contributions du R.P. Dahin ont été suivies d'une publication modeste en un volume. Il s'agit d'un dictionnaire de poche du yinzebi intitulé Petit dictionnaire bantou du Gabon: français-ndjabi; ndjabi-français produit par Muroni (1989). Sur la même langue, Nadaillac a produit un Lexique yinzebi-français en collaboration avec l'équipe de l'ALGAB. Le dictionnaire incluant le yinzebi et le français comme langues paires est en préparation. Enfin, l'ouvrage lexicographique le plus important de ce groupe est certainement le Dictionnaire ndumu-mbede-français par Biton et Adam (1969). Cet ouvrage de Biton et Adam est intéressant parce qu'il n'implique pas que le français et l'un des parlers du Gabon comme c'est le cas dans la plupart des dictionnaires existants, mais il implique à la fois deux langues du Gabon (liduma et lateghe) et le français. Les sources primaires de ce dictionnaire multilingue sont probablement la Grammaire composée mbede-ndumu-duma qu'Adam a publié en 1954. Cependant, ce livre de grammaire doit être considéré comme seulement une des diverses sources de l'auteur. En effet, après 52 ans au Gabon - un pays où il s'est éteint en 1981 - la contribution d'Adam à la littérature orale gabonaise inclut plusieurs articles sur les différents parlers de la province du Haut-Ogooué. Ce dictionnaire de Biton et Adam constitue également une passerelle entre certaines langues du groupe dit metye et celles du groupe membere qui fait l'objet de la section suivante.

\section{Le Groupe Membere}

Les parlers de ce groupe sont principalement localisés dans la province du Haut-Ogooué. Le lembaama est parlé dans les régions de Franceville-OkondjaAkiéni, le lekanini à Franceville et à Boumango, le lateghe à Akiéni et à Léconi et le latsitseghe à Léconi. Le lindumu est utilisé à Franceville.

La littérature disponible sur ce groupe inclut des travaux religieux, des études de littérature orale ou encore à caractère ethnohistorique ainsi qu'un certain nombre de travaux linguistiques. Nous mettrons ici l'accent sur les travaux linguistiques.

Les travaux linguistiques dans ce groupe apparaissent déjà dans les premières études lexicographiques. Les travaux de Biton (1907) et Biton et Adam (1969) sont certes des travaux lexicographiques mais ils sont précédés d'une description grammaticale des langues faisant l'objet de l'étude. Les travaux réalisés dans le cadre de la linguistique moderne incluent les contributions des auteurs suivants sur le lateghe, le lekanini, le lembaama et le lendumu respectivement: Puech (1990), Kwenzi-Mikala (1990), Alihanga, Teta et Voltz (1990) et enfin Binga et Puech (1990). Les contributions de Binga et Puech sur le lendumu ont été précédées par un certain nombre de travaux: Mouélé (1988) et 
Medjo-Mvé (1989). La contribution de Kwenzi-Mikala sur le lekanini a été suivie quelques années plus tard par le travail d'Evouna Ntoutoume (2003). Tous ces travaux présentent à un degré plus ou moindre les éléments de phonologie et de morphologie des langues décrites. À ce titre, ils gagneraient à être pris en compte par les lexicographes travaillant sur lesdites langues.

La lexicographie membere commence avec la publication du dictionnaire biscopal intitulé Dictionnaire français-ndumu et ndumu-français par le R.P. Biton (1907). L'ouvrage couvre 97 pages et est divisé en deux parties. La première partie présente au lecteur la grammaire du lindumu, tandis que la deuxième partie contient le dictionnaire à proprement parler. La première section du dictionnaire commence par des lemmes en français. Ensuite, le lexicographe renverse les langues dans la deuxième section du dictionnaire. Le dictionnaire en question a servi comme point de départ pour la production de matériel didactique religieux tels que le Kateçism Ndumu (1962) et quelques années plus tard, le Dictionnaire ndumu-mbede-français et français-ndumu-mbede produit par Biton en collaboration avec Adam en 1969. Pour rappel, la caractéristique de ce dictionnaire est qu'il implique trois langues (le français, lindumu (ndumu) et lateghe (mbede)) et que les langues sont renversées dans le seul et même dictionnaire. En outre, les lemmes sont extraits du fonds lexical des noms de plantes de la région de Franceville. Le prochain travail à examiner est un lexique interdialectal intitulé "Vocabulaire comparé des principaux dialectes ayant cours en Haut-Ogooué" publié par Castex en 1938. L'ouvrage est une contribution intéressante sur le domaine de la dialectologie (également appelé géographie linguistique). Par exemple, il pourrait être utilisé comme un travail de référence valable pour la production d'un dictionnaire multilingue ou pandialectal impliquant les différents parlers du groupe membere. Par rapport au groupe membere, les unités-langues mekana et baka quoique présentant une littérature lexicographique quasi inexistante ont de riches perspectives de développement.

\section{Le Groupe Mekana}

Les parlers de ce groupe sont principalement localisés dans la province l'Ogooué-Ivindo. Le bekwil (ou bekwel) est parlé à Makokou, le shiwa (ou makina) à Booué et le mwẹsa (ou yẹsa) à Mékambo. En ce qui concerne ce groupe, il reste encore beaucoup à faire. Cependant, les contributions de Puech (1990), Yembi Bouka (1995), Kwenzi-Mikala (1996: 10-16) et Mouloungui (1999) contiennent un aperçu de la grammaire et de la littérature orale du shiwa, du bekwel et du mwesa respectivement. En ce qui concerne les publications lexicographiques, rien n'est pour le moment disponible.

\section{Le Groupe Baka}

Le baka est la seule langue de ce groupe. Il est parlé à Minvoul, à Makokou et à 
Belinga. La littérature disponible dans ce groupe inclut le travail de Mayer (1987) et de Mayer et Voltz (1990) dans le domaine de l'anthropologie, entre autres. Les activités lexicographiques restent à entreprendre.

\section{En guise de conclusion}

L'état des connaissances ci-dessus sur les travaux religieux, ethnohistoriques, linguistiques et lexicographiques sur les langues gabonaises montre clairement et de façon générale l'existence d'une riche littérature. Toutefois, une vue d'ensemble de cette littérature fait également apparaître des déséquilibres entre les langues et les unités-langues. Certaines sont de loin plus avancées que d'autres. Le traitement de chaque groupe varie en importance selon les données religieuses, ethnohistoriques, linguistiques et lexicographiques disponibles.

Ces dernières lignes ne sauraient à proprement parler constituer une conclusion. Si la parution de cet article suscite dans le pays une coopération beaucoup plus poussée entre chercheurs d'origines et de formations diverses (linguistes, sociologues, historiens, lexicographes, etc.), il aura gagné son pari. Ce propos est motivé par le constat suivant de Mavoungou (À paraître):

La majorité des sources de référence lexicographique existant dans les langues gabonaises auxquelles nous avons fait allusion plus haut ont requis des efforts considérables et de l'abnégation si on considère le fait que la plupart de ces travaux était le résultat d'une vie de travail par des auteurs isolés. Comme pour toute œuvre pionnière, ces dictionnaires, lexiques, encyclopédies de la première heure comportent des lacunes tant au niveau linguistique que métalexicographique. Cependant, ces manquements sont excusables si l'on considère que les lexicographes de ces travaux précurseurs n'étaient ni linguistes, ni lexicographes qualifiés.

Les travaux lexicographiques sur les langues gabonaises publiés en ce début de troisième millénaire devraient être des ouvrages collectifs, c'est-à-dire le fruit d'un travail d'équipe, nouveau défi, dans ce monde qui ne cesse de se mondialiser.

\section{Notes}

1. Dans cet article l'orthographe officielle du Gabon est utilisée en lieu et place de la traditionnelle transcription semi-phonétique par Guthrie, Kwenzi-Mikala, et les autres. Les $\underline{e}, \underline{o}$ et $\underline{n}$ souscrits représentent $[\varepsilon]$, [o] et $[\eta]$ respectivement, tandis que $[\gamma],[J]$ et [ $[\beta]$ sont écrits gh, sh et vh respectivement. Le son phonétique [ə] est représenté par le symbole a dans l'orthographe gabonaise. Toutefois pour des raisons phonologiques et pratiques, il est représenté par a comme dans Make et Ghisira.

2. Selon la tradition du mot, les items lexicaux sont présentés sous leur forme entière, par exemple leur préfixe plus le thème. Par contre, dans la tradition du thème les lexèmes sont lemmatisés sous le thème sans leurs préfixes. 
3. Dans les notes introductives du DGF (2002: 4), les auteurs soulignent qu'"on peut estimer le nombre de bons locuteurs à une quarantaine seulement. Ce chiffre extrêmement bas indique sans ambiguïté aucune que le geviya est une langue dont la disparition est sur le point de s'achever."

4. Atlas Linguistique du Gabon. La réalisation de cet atlas échoit aux chercheurs du Laboratoire de Phonétique et de Linguistique Africaine (LAPHOLIA) de l'Université Lumière Lyon 2 sous la direction de Jean-Marie Hombert.

\section{Références bibliographiques}

Adam, J. 1954. Grammaire composée mbede-ndumu-duma. Brazzaville: Institut d'Étude Centrafricaines.

Afane Otsaga, T. 2004. The Standard Translation Dictionary as an Instrument in the Standardization of Fang. Thèse de Doctorat non publiée. Stellenbosch: Université de Stellenbosch.

Agondjo-Okawe, P. 1967. Structures parentales gabonaises et développement. Thèse de Doctorat non publiée. Paris: Université de Paris I.

Alexandre, P. 1961. Problèmes linguistiques des états négro-africains à l'heure de l'indépendance. Cahiers d'études africaines 2(6): 177-195.

Alexandre, P. 1967. Langues et langage en Afrique Noire. Paris: Payot.

Alihanga, M., R. Teta et M. Voltz. 1990. Lembaama. Revue Gabonaise des Sciences de l'Homme 2: 235241.

Ambouroue-Avaro, J. 1981. Un peuple gabonais à l'aube de la colonisation. Présence Africaine. Paris: Karthala.

Andeme Allogho, M.F. 1983. Esquisse phonologique du Nzaman de Makokou. Mémoire de Licence non publié. Libreville: Université Omar Bongo.

Avelot, R. 1911. Notes sur les pratiques religieuses des Bakalè. Bulletin et Mémoire de la Société d'Anthropologie de Paris 6: 282-296.

Balandier, G. 1957. Afrique ambiguë. Paris: Plon.

Balandier, G. 1963. Sociologie actuelle de l'Afrique Noire. Paris: PUF.

Bibang Meye. 2001. Étude des compétences narratives chez les enfants bilingues Fang-Ntumu-Français d'Oyem (psycholinguistique): Étude transversale sur le développement de la temporalité et de la connectivité chez des sujets âgés de 7 à 12 ans. Mémoire de Maîtrise. Libreville: Université Omar Bongo.

Binga, H. et G. Puech. 1990. Lendumu. Revue Gabonaise des Sciences de l'Homme 2: 243-246.

Biton, A. (R.P.). 1903. Cantiques endumu-ambété-akota-alimi. Mission Catholique de BrazzavilleFranceville. Nantes: Imprimerie Bourgeois.

Biton, A. (R.P.). 1907 Dictionnaire français-ndumu et ndumu-français, précédé d'éléments de grammaire. Nantes: Imprimerie Dupas.

Biton, A. (R.P.). 1962. Kateçism Ndumu. Rome: Société St Pierre Claver.

Biton, A. (R.P.) et J. Adam (Mgr.). 1969. Dictionnaire ndumu-mbede-français et français-ndumumbede. Petite flore de la région de Franceville (Gabon). Grammaire ndumu-mbede. Archevêché de Libreville. Bar-le-Duc: Imprimerie St Paul.

Blanchon, A. 1988. Tonalité des nominaux à thème dissyllabique dans le groupe bantou B20. Pholia. Revue du Laboratoire de Phonétique et Linguistique Africaine 3: 37-52. 
Blanchon, J.A. 1988a. Une langue mixte en voie de disparition: le geviya. Pholia. Revue du Laboratoire de Phonétique et Linguistique Africaine 3: 53-69.

Blanchon, J.A. 1997. Les formes nominales de citation à préfixe haut en pounou (Bantu B43). Journal of African Languages and Linguistics 18(2): 129-138.

Blanchon, J.A. 1997a. Dictionnaire informatisé pounou-français. Manuscrit. Lyon: Université Lumière Lyon 2.

Bodinga-bwa-Bodinga, S. 1969. Traditions orales de la race eviya. Paris: T.M.T.

Bodinga-bwa-Bodinga, S. et L.J. van der Veen. 1993. Plantes utiles des Evia. Pholia. Revue du Laboratoire de Phonétique et Linguistique Africaine 8: 26-65.

Bodinga-bwa-Bodinga, S. et L.J. van der Veen. 1995. Les proverbes evia et le monde animal, la communauté evia à travers ses expressions proverbiales. Paris: L'Harmattan.

Bonneau, J. (R.P.). 1956. Grammaire pounoue et lexique pounoue-français. Brazzaville: Institut d'Études Centrafricaines.

Buléon, J. (R.P.). 1887. Le lac et la tribu des Nkomi. Annales de la Propagation de la Foi: 175-182.

Buléon, J. (R.P.). 1892. Le lac et la tribu des Nkomi. Annales de la Propagation de la Foi: 437-448.

Buléon, J. (R.P.). 1899. Catéchisme de la Doctrine Catholique en langue Eshira. Paris: Mission Catholique de Libreville (St Croix).

Castex (Dr.). 1938. Vocabulaire comparé des principaux dialectes ayant cours en Haut-Ogooué. Essai de classification. Bulletin de la Société des Recherches Congolaises 26: 23-54.

Chela, P. 1998. Étude des emprunts linguistiques du nzébi, langue bantu du Gabon (B52), au français; procédés d'integration. Mémoire de Maîtrise. Libreville: Université Omar Bongo.

Cinnamon, J. 1990. Fan (nzaman). Revue Gabonaise des Sciences de l'Homme 2: 175-183.

Dahin (R.P.). 1891. Catéchisme en langue adouma (Haut-Ogooué) = Mambo ma ndjambi ghu evovili si a tshenge a baduma. Rixheim: Imprimerie A. Sutter.

Dahin (R.P.). 1893. Vocabulaire français-aduma. Kempten (Bavière): Kosel.

Dahin (R.P.). 1895. Vocabulaire aduma-français. Kempten (Bavière): Kosel.

Delorme, A. 1877. Dictionnaire français-mpongwè. Paris: Missionnaires de la Congrégation du St Esprit et du St Cœur de Marie.

Derouet (R.P). n.d. Dictionnaire français-vili. Loango: Imprimerie de la Mission.

Deschamps, H. 1962. Traditions orales et archives au Gabon. Paris: Berger-Levrault.

Dodo-Bounguedza, E. 1986. Perspectives linguistiques pour l'enseignement du Liwandji (cours préparatoires première et deuxième année). Mémoire de Maîtrise. Libreville: Université Omar Bongo.

Dodo-Bounguedza, E. 1992. Esquisse phonologique et morphologique du gisira, langue bantoue (B41) du Gabon. Thèse de Doctorat non publiée. Bruxelles: Université Libre de Bruxelles.

Ella, E.M. 2000. Étude linguistique d'une oeuvre littéraire: Un mvet de Zué Nguema, chant épique Fang recueilli par Herbert Pepper. Mémoire de Maîtrise. Libreville: Université Omar Bongo.

Ella Ella, E. 2000. Étude contrastive des pronoms relatifs en Français et en Ntumu. Mémoire de Maîtrise. Libreville: Université Omar Bongo.

Essono, E. 1988. Esquisse phonologique et morphologique du Benga, langue bantu du Gabon. Mémoire de Maîtrise. Libreville: Université Omar Bongo.

Essono, E. 1990. Benga. Revue Gabonaise des Sciences de l'Homme 2: 129-139.

Évangile de Jean: traduction dans la langue des Fan. 1910. Paris.

Évangile de Luc: traduction dans la langue des Fan. 1910. Paris.

Évangile de Matthieu: traduction dans la langue des Fan. 1902. Paris. 
Even, A. 1937. Les confréries secrètes chez les Babamba et les Mindassa d'Okondja. Bulletin de la Société des Recherches Congolaises 23: 31-133.

Evouna Ntoutoume, F. 2003. Description phonologique du lekanini (langue bantoue du Gabon B.66). Mémoire de Maîtrise. Libreville: Université Omar Bongo.

Gachon, J.-B. 1881. Dictionnaire mpongwè-français, précédé des principes de la langue mpongwè. Paris.

Gachon, J.-B. 1891. Bible gnango ine agamba mi re tendo pa gou'ejango j'agnambie ji felio né TESTAMAN NOUNGOU NI TESTAMAN GNONA gou'inongo gni mpongoue. Sente-Maria yi Gabon. Fribourg-enBrisgau: Herber B. Libr. Edit. Pontifical.

Galley, S. 1889. Nteni osi nteni Fañwe. Talagouga: Imprimerie de la Mission.

Galley, S. 1964. Dictionnaires Fang-Français et Français-Fang, suivi d'une grammaire Fang. Neuchâtel: Henri Messeiller.

Gamille, G. 1997. Le Mungala entre le Congo et le Gabon: Étude sociolinguistique de la variation d'un récit mythique. Rapport de Licence. Libreville: Université Omar Bongo.

Garnier, A. 1897. Katesisa i gheghe nesi ma loghi ma dzambi mu mbembu i-lumbu. Loango: Imprimerie de la Mission.

Garnier, A. 1900. Syllabaire i-lumbu keti mi ganda mio mi teti mi ranganga mu mbembo i lumbu. Loango: Imprimerie de la Mission.

Garnier, A. 1903. Mu mbembo kivili ki mayumbe. Loango: Imprimerie de la Mission.

Garnier, A. 1904. M'ambu ma nzambi mo make mu katesisa. Loango: Imprimerie de la Mission.

Gaulme, F. 1976. L'ancien pays de Cama, sociéte et organisation politique du XVIe au XIXe siècle. Thèse de Doctorat. Paris: Université de Paris V.

Gaulme, F. 1981. Le pays de Cama. Un ancien État-côtier du Gabon et ses origines. Paris: Karthala.

Gautier, D. (R.P.). 1950. Étude historique sur les Mpongoués et les tribus avoisinantes. Brazzaville: A.E.F.

Gregoire, C. 1990. Omyene (nkomi). Revue Gabonaise des Sciences de l'Homme 2: 273-280.

Grimes, B.F. (Éd.) 1996 ${ }^{13}$. Ethnologue: Languages of the World. Dallas: Summer Institute of Linguistics.

Guthrie, M. 1948. The Classification of the Bantu Languages. Londres: Oxford University Press.

Guthrie, M. 1953. The Bantu Languages of Western Equatorial Africa. Londres: Oxford University Press.

Guthrie, M. 1969-1971. Comparative Bantu: An Introduction to the Comparative Linguistics and Prehistory of the Bantu Languages. 5 tomes. Farnborough: Gregg International.

Haug, E. 1903. Le Bas-Ogoué. Notice géographique et ethnographique. Annales de géographie 12: 169-171.

Hauser, A. 1954. Notes sur les Omyéné du Bas-Gabon. Bulletin de l'Institut Français d'Afrique Noire (BIFAN) 16(3-4): 402-415.

Hombert, J.-M. 1990. Ikota. Revue gabonaise des Sciences de l'Homme 2: 211-212.

Hombert, J.-M. et A.-M. Mortier. 1984. Mise à jour de la bibliographie publiée dans Pholia. Revue du Laboratoire de Phonétique et Linguistique Africaine 1: 165-187.

Hombert, J.-M. et A.-M. Mortier. 1990. Bibliographie des langues du Gabon. Revue gabonaise des Sciences de l'Homme 2: 335-355.

Idiata, D.F. 1989. Éléments de phonologie du yepinzipinzi. Mémoire de Maîtrise. Libreville: Université Omar Bongo. 
Idiata, D.F. 1993. Éléments de phonologie diachronique du isangu, correspondances Proto-Bantu-Isangu et lexique spécialisé. Mémoire de D.E.A. Lyon: Université Lumière Lyon 2.

Idiata, D.F. 1998. Quelques aspects de l'acquisition de la langue isangu par les enfants. Vol. 1: Étude des processus d'acquisition; Vol. 2: Les protocoles expérimentaux; Vol. 3: Esquisse de description grammaticale de la langue isangu. Thèse de Doctorat (NR). Lyon: Université Lumière Lyon 2.

Jacot, H. 1894. Ezangô elônga zi mpôngwe gw'itangula nl'itenda. Premier livre de lecture en mpongwè. New York: Société américaine des Traités Religieux.

Jacquot, A. 1978. Le Gabon. Barreteau, D. (Éd.). 1978. Inventaire des études linguistiques sur les d'Afrique noire d'expression française et sur Madagascar: 493-503. Paris: CILF.

Jacquot, A. 1983. Les classes nominales dans les langues bantoues des groupes B10, B20, B30 (GabonCongo). Travaux et documents de l'ORSTROM 157. Paris: ORSTROM.

Kane, F. 1998. Étude contrastive du français et du mponwè. Approche phonétique et phonologique. Mémoire de Maîtrise. Libreville: Université Omar Bongo.

Koumba, V. 1990. Essai de phonologie du gisir (langue bantu du Gabon B41). Mémoire de Maîtrise. Libreville: Université Omar Bongo.

Kwenzi-Mikala, J.T. 1988. L'identification des unités-langues bantu gabonaises et leur classification internes. Muntu 8: 54-64.

Kwenzi-Mikala, J.T. 1989. Contribution à l'analyse des emprunts nominaux du yipunu au français. Pholia. Revue du Laboratoire de Phonétique et Linguistique Africaine 4: 157-170.

Kwenzi-Mikala, J.T. 1990. Lekanini. Revue Gabonaise des Sciences de l'Homme 2: 227-234.

Kwenzi-Mikala, J.T. 1990a. L'anthroponymie chez les Bapunu du Sud-Gabon. Pholia. Revue du Laboratoire de Phonétique et Linguistique Africaine 5: 113-120.

Kwenzi-Mikala, J.T. 1996. Parémies d'Afrique Centrale (Proverbes et Sentences). Libreville: Éditions Raponda-Walker.

Kwenzi-Mikala, J.T. 1998. Parlers du Gabon: classification du 11.12.97. Raponda-Walker, A. (Éd.). 1998: 217.

Lamour (R.P.). 1936. Petit catéchisme ikota. Manuscrit. Libreville.

Largeau, V. 1901. Encyclopédie pahouine. Eléments de grammaire et dictionnaire français-pahouin. Paris: E. Leroux.

Lejeune, L. (R.P.) 1892. Dictionnaire français-fang ou pahouin, précédé de quelques principes grammaticaux sur cette même langue. Paris: A. Faivre/H. Teillard.

Lemoine, G.-M. (Sœur). 1994. Petit dictionnaire français-gisira-ngosi et éléments de verbes. Libreville: Mission Sainte-Croix de Mandji-Ndolou.

Mabika Mbokou, L. 1999. Les phénomènes analogiques en civili: Étude phonologique et morphologique. Mémoire de Maîtrise. Libreville: Université Omar Bongo.

Mackey, J.L. 1855. Grammar of the Benga Language. New York: Mission House.

Makoungou, E. 1999. L'étude comparée du lexique du français et des parlers de l'unité-langue metye; le yinzébi. Mémoire de Maîtrise. Libreville: Université Omar Bongo.

Manfoumbi, M. 1989. Eléments de phonologie du Saké í mèmbónzù dialecte saké de Booué. Mémoire de Maîtrise. Libreville: Université Omar Bongo.

Manfoumbi, M. et M.-E. Zambibonda. 1990. Jake. Revue Gabonaise des Sciences de l'Homme 2: 287290.

Marchal-Nasse, C. 1979. Esquisse de la langue tsogo: phonologie et morphologie. Mémoire de Licence Spéciale. Tervuren: Université Libre de Bruxelles. 
Marchal-Nasse, C. 1989. De la phonologie à la morphologie du nzebi, langue bantoue (B52) du Gabon. Thèse de Doctorat de 3ième cycle. Tervuren: Université Libre de Bruxelles.

Marchal-Nasse, C. 1990. yetsogo. Revue Gabonaise des Sciences de l'Homme 2: 185-188.

Marchal-Nasse, C. 1990a. Yinzebi. Revue Gabonaise des Sciences de L'Homme 2: 295-298.

Marichelle, C. 1902. Dictionnaire français-vili. Loango: Imprimerie de la Mission.

Marling (R.P.). 1872. Dictionnaire fang-français. New York.

Martrou, L. 1924. Lexique fãn-français. Paris: Procure Générale (des Pères du St Esprit)/Abbeville: Imprimerie Paillard.

Mavoungou, P.A. 1998. Esquisse phonologique et morphologique du nda fa parlé à Mandjaye. Mémoire de Maîtrise. Libreville: Université Omar Bongo.

Mavoungou, P.A. 2001. Macro- and Microstructural Issues in Mazuna Lexicography. Lexikos 11: 122-138.

Mavoungou, P.A. 2001a. La mondialisation et la lexicographie trilingue ou plurilingue au Gabon. Emejulu, J.D. (Éd.). 2001. Éléments de lexicographie gabonaise Tome I: 160-183. New York: Jimacs-Hillman.

Mavoungou, P.A. 2002. Metalexicographical Criteria for the Compilation of a Trilingual Dictionary: Yilumbu-English-French. Thèse de Doctorat non publiée. Stellenbosch: Université de Stellenbosch.

Mavoungou, P.A. 2004. L.J. van der Veen et Bodinga-bwa-Bodinga: Gedandi sa Geviya/Dictionnaire Geviya-Français. Lexikos 14: 440-448.

Mavoungou, P.A. À paraître. Lexicographie et confection des dictionnaires au Gabon. Stellenbosch: SUN PreSS.

Mavoungou, P.A., T. Afane Otsaga et G.-R. Mihindou. 2003. The Reproduction of Cultural Aspects in Dictionaries in French and the Gabonese Languages. Lexikos 13: 133-153.

Mayer, R. 1987. Langues des groupes Pygmées du Gabon: un état des lieux. Pholia. Revue du Laboratoire de Phonétique et Linguistique Africaine 2: 119-127.

Mayer, R. 1990. Histoire de l'écriture des langues du Gabon. Revue Gabonaise des Sciences de l'Homme 2: 65-91.

Mayer, R. et M. Voltz. 1990. Dénominations ethnoscientifiques des langues et des ethnies du Gabon. Revue Gabonaise des Sciences de l'Homme 2: 43-53.

Mba-Nkoghe, J. 1981. Phonologie et classes nominales en fang (Langue bantoue de la zone A). Thèse de 3ième cycle. Paris: Université Sorbonne Nouvelle.

Mba-Nkoghe, J. 1991. Place et utilité des langues gabonaises dans l'activité nationale. Bulletin d'information et de liaison du Ministère de l'Education Nationale. Message 5: 18-22.

Mba-Nzué, N. 1981. Esquisse phonologique du mvaï (Parler de Minvoul). Mémoire de Licence. Libreville: Université Omar Bongo.

Mbougou Asseko, M. 1979. Étude contrastive du Fang d'Oyem et du Fang de Libreville. Mémoire de Licence. Libreville: Université Omar Bongo.

Medjo-Mvé, P. 1989. Exploration morpho-syntaxique du dialecte le nyani ou contribution à l'étude du verbe ndumu. Mémoire de Maîtrise. Libreville: Université Omar Bongo.

Medjo-Mvé, P. 1997. Essai sur la phonologie panchronique des parlers fang du Gabon et ses implications historiques. Thèse de Doctorat (NR). Lyon: Université Lumière-Lyon 2.

Meinhof, C. 1889-1890. Das Zeitwort in der Benga-Sprache. Zeitschrift für afrikanische Sprachen 3: 265-284. 
Mekui Missang, E. 1998. La présence des faits suprasegmentaux du fang ntumu dans l'utilisation du français pour les enfants âgés de 10 à 12 ans. Mémoire de Maîtrise. Libreville: Université Omar Bongo.

Mickala-Manfoumbi, R. 1986. Une approche linguistique pour l'enseignement du yipunu. Mémoire de Maîtrise. Libreville: Université Omar Bongo.

Mickala-Manfoumbi, R. 1989. Éléments de description du duma, langue bantu du Gabon (B51). Mémoire de Licence Spéciale. Bruxelles: Université Libre de Bruxelles.

Mickala-Manfoumbi, R. 2004. Lexique pove-français/français-pove. Libreville: Éditions RapondaWalker.

Mission Catholique de Libreville. 1948. Ezango zi chrétien mpongze-orungu-galoa-nkomi. Issy-LesMoulineaux: Presses Missionnaires Imprimerie St Paul.

Mission Catholique de Libreville. Vicariat Apostolique de Libreville. 1894. I kambisa si kretien = Livre de prières en mpongzè-français-latin. Libreville: Vicariat Apostolique du Gabon.

Mission Catholique de Sette-Cama. 1933. Catéchisme de la doctrine chrétienne = Katsisu mu mbembo hi lumbu hi bis'sette-cama. Paris: Imprimerie des Orphelins Apprentis d'Auteuil.

Mission Catholique du Gabon. Vicariat Apostolique du Gabon. 1925. Syllabaire Fan. Merville (Nord): Imprimerie Ceugnard Le Sage.

Mission Catholique du Gabon. Vicariat Apostolique du Gabon. 1932. Catéchisme Fan. Rome: Solidarité St Pierre Claver.

Mission de la Sainte-Croix. 1924. Catéchisme Gisira. Abbeville: Imprimerie Paillart.

Mouélé, M. 1988. Description d'un parler Ndumu le lenyani (phonétique, phonologie, morphologie nominale et pronominale). Mémoire de Maîtrise. Libreville: Université Omar Bongo.

Mouélé, M. 1997. Étude synchronique et diachronique du groupe duma. Thèse de Doctorat (NR). Lyon: Université Lumière Lyon 2.

Mouguiama-Daouda, L. 1991. Étude morpho-syntaxique du constituant nominal en ygósì, dialecte du gisira. Mémoire de D.E.A. Lyon: Université Lumière Lyon 2.

Mouguiama-Daouda, P. 1995. Les dénominations ethnoichtyologiques chez les bantous du Gabon. Thèse de Doctorat (NR). Lyon: Université Lumière Lyon 2.

Mouloungui, M.P. 1999. Esquisse phonologique et morphologique du yesa. Mémoire de Maîtrise. Libreville: Université Omar Bongo.

Murard, P. (Trad.). 1903. Katsisu i neni i rendilu mu mbembo bis'Sette-Cama (grand catéchisme). Lyon: Imprimerie Paquet.

Murard, P. (Trad.). 1903a. Katsisu i keki i rendilu mu mbembo bis'Sette-Cama (petit catéchisme). Lyon: Imprimerie Paquet.

Murard, P. (Trad). 1903b. Cantiques = Gnimbu tsi niambi. Lyon: Imprimerie Paquet.

Muroni, J.-M. 1989. Petit dictionnaire bantou du Gabon: français-ndjabi, ndjabi-français. Paris: L'Harmattan.

Naidaillac, L. 1992. Lexique isangu-français. Inédit. Lyon: Université Lumière Lyon 2.

Naidaillac, L. 1992a. Lexique yinzebi-français. Inédit. Lyon: Université Lumière Lyon 2.

Nassau, R.H. 1881. Panwe Primer and Vocabulary Compiled from Materials Collected by H.M. Adam. New York: American Tract Society.

Ndamba, J. 1977. Syntageme nominal et groupe nominal en vili: Langue bantu du Congo. Thèse de Doctorat 3ième cycle. Paris: Université de Paris III Sorbonne Nouvelle. 
Ndinga-Koumba-Binza, H.S. 2000. Phonologie du civili de Mayumba: Langue bantu du Gabon (H12a). Mémoire de Maîtrise. Libreville: Université Omar Bongo.

Ndong, F. 1962. Nten Wam. Rome: Société St Pierre Claver.

Ndong Menini, I. 1980. Esquisse phonologique du ntumu. Mémoire de Licence. Libreville: Université Omar Bongo.

Ndongo Esono, S. (R.P.). 1956. Gramática pamue. Madrid: Instituto de Estudios Africanos.

Ndoumou Mba. 1999. Étude diachronique des phonèmes du yinzebi et du proto-bantu. Mémoire de Maîtrise. Libreville: Université Omar Bongo.

Nguema Nzue, R. 1988. Essai de morpho-syntaxe du Fang-Ntumu. Mémoire de Licence. Libreville: Université Omar Bongo.

Nsuka-Nkutsi, F. (Éd.). 1980. Éléments de description du Punu. Lyon: Presses Universitaires de Lyon (PUL).

Nyangone Assam, B. et P.A. Mavoungou. 2000. Lexicography in Gabon: A Survey. Lexikos 10: 252274.

Nzang-Obame, J.E. 2001. Essai de description phonologique de fang-mekè (A75). Mémoire de Maîtrise. Libreville: Université Omar Bongo.

Nzang-Bié, Y. 1986. Quelques aspects de la technique du contage chez les Fang Ntumu. Mémoire de Licence. Libreville: Université Omar Bongo.

Ondo Essono Aba'a. 1981. Esquisse morphologique du Ntumu. Mémoire de Licence. Libreville: Université Omar Bongo.

Ondo-Mebiame, P. 1989. Esquisse de description du sangu, langue bantu du Gabon de zone B42. Mémoire de Licence Spéciale. Bruxelles: Université Libre de Bruxelles.

Ondo-Mebiame, P. 1992. De la phonologie à la morphologie du Fang parlé à Aboumezok (Langue Bantu A.78). 2 volumes. Thèse de Doctorat (NR). Tervuren: Université Libre de Bruxelles.

Ondo-Mebiame, P. et Y. Nzang-Bié. 1990. Scki. Revue Gabonaise des Sciences de l'Homme 2: 281-286.

Osorio, Z. 1887. Diccionario Pamue-Español, Vocabulary of the Fan Language in Western Africa. London.

Perez, G. et L. Sorinas. 1928. Gramática de la lengua Benga. Madrid: Editorial del Corazón de María.

Perron, H.P. (R.P.). 1964. Lexique français-ikota. 2 vol. (reprise des notes manuscrites du R.P. Lamour). Makokou: Mission Catholique.

Puech, G. 1990. Lateye. Revue Gabonaise des Sciences de l'Homme 2: 213-226.

Puech, G. 1990. Bekwel. Revue Gabonaise des Sciences de l'Homme 2: 127-128.

Raponda-Walker, A. (n.d.) Dictionnaire getsogo-français/français-getsogo. Manuscrit inédit.

Raponda-Walker, A. 1930-1934. Dictionnaire mpongwè-français, suivi d'éléments de grammaire. Metz: Imprimerie de la Libre Lorraine.

Raponda-Walker, A. 1937. Initiation à l'ébongwè, langues des Negrilles. Bulletin de la Société des Recherches Congolaises (BRC) 23: 129-156.

Raponda-Walker, A. 1961. Dictionnaire français-mpongwé. Brazzaville: Imprimerie St Paul. 19952. Classiques Africains. Libreville: Éditions Raponda-Walker.

Raponda-Walker, A. 1995. Dictionnaire mpongwe-français. Versailles/Libreville: Classiques Africains/Éditions Raponda-Walker.

Raponda-Walker, A. 1995. Dictionnaire français-mpongwe. Versailles/Libreville: Classiques Africains/Éditions Raponda-Walker. 
Raponda-Walker, A. (Éd.). 1998. Les langues du Gabon. Classiques Africains. Libreville: Éditions Raponda-Walker.

Raponda-Walker, A. et R. Sillans. 1961. Les plantes utiles du Gabon: essai d'inventaire et de concordance des noms vernaculaires et scientifiques des plantes spontanées et introduites du Gabon. Paris: Lechevalier.

Ratanga-Atoz, A.F. 1999. Les peuples du Gabon Occidental de 1839-1941. Tome 1. Libreville: Éditions Raponda-Walker.

Reeb, A. 1895. Bivovili bya bakretyeni ba baduma = Manuel de prières à l'usage des chrétiens doumas. Mission Catholique de Libreville-Lastoursville. Fribourg-en-Brisgau: Imprimerie par Herder.

Rekanga, J.P. 1986. Pour une lecture morpho-syntaxique du chant II du Mvet de Zué Nguéma. Mémoire de Licence. Libreville: Université Omar Bongo.

Rittaud-Hutinet, C. 1980. Lexique. Nsuka-Nkutsi, F. (Éd.). 1980. Éléments de description du Punu: 193-245. Lyon: Presses Universitaires de Lyon (PUL).

Sima Mvé, J.B. 1996. Esquisse phonologique et morphologique du mahongze. Rapport de Licence. Libreville: Université Omar Bongo.

Tardy, L. 1933. Contribution à l'étude du folklore bantou: les fables, devinettes et proverbes fang. Anthropos 28(3-4): 277-303.

Tchen-Damas, E. 1990. Omyene (mpongwe). Revue Gabonaise des Sciences de l'Homme 2: 261-271.

Trilles, H. 1898. Livre de prières = NTEN WAM minkobe ma nè ge kobe né nzame. Mission catholique de Libreville. Bar-le-Duc: Imprimerie de l'œuvre St Paul.

Trilles, H. 1898. Ntèn wa tar'éyé gele fang'ne fala étén nélang = Exercices de lecture et d'écriture en pahouin et en français. (2e partie). Mission Catholique de Libreville. Tours: Imprimerie Louis Dubois.

Trilles, H. n.d. Katesism Nten wa vegele Fang Nsong Katolik = Catéchisme de la Doctrine Catholique. Paris: CSSP et Mission Catholique de Libreville.

Van der Veen, L.J. 1991. Étude comparée des parlers du groupe Okani - B30 (Gabon). Thèse de Doctorat (NR). Lyon: Université Lumière-Lyon 2.

Van der Veen, L.J. 1992. Le système tonal du ge-via (Gabon). Journal of West African Languages 22(2): 17-41.

Van der Veen, L.J. 1999. Les Bantous eviya (Gabon-B30): langue et société traditionnelle. Note de synthèse en vue de l'obtention de l'Habillitation à Diriger des Recherches en Sciences du Langage, soutenue le 28 janvier 1999 à l'Université Lumière-Lyon 2.

Van der Veen, L.J. et S. Bodinga-bwa-Bodinga. 2002. Gedandedi sa Geviya/Dictionnaire GeviyaFrançais. Louvain/Paris/Sterling, Virginia: Peeters.

Yembi Bouka, L. 1995. Structures phonologiques et structures prosodiques (le modèle bekwel). Thèse de Doctorat (NR). Tervuren: Université Libre de Bruxelles. 